\title{
Simulation of ultrafast excited-state dynamics and elastic x-ray scattering by quantum wavepacket dynamics
}

Pápai, Mátyás Imre; Rozgonyi, Tamás; Penfold, Thomas J.; Nielsen, Martin Meedom; Møller, Klaus Braagaard

Published in:

Journal of Chemical Physics

Link to article, DOI:

$10.1063 / 1.5115204$

Publication date:

2019

Document Version

Publisher's PDF, also known as Version of record

Link back to DTU Orbit

Citation (APA):

Pápai, M. I., Rozgonyi, T., Penfold, T. J., Nielsen, M. M., \& Møller, K. B. (2019). Simulation of ultrafast excitedstate dynamics and elastic $x$-ray scattering by quantum wavepacket dynamics. Journal of Chemical Physics, 151(10), [104307]. https://doi.org/10.1063/1.5115204

\section{General rights}

Copyright and moral rights for the publications made accessible in the public portal are retained by the authors and/or other copyright owners and it is a condition of accessing publications that users recognise and abide by the legal requirements associated with these rights.

- Users may download and print one copy of any publication from the public portal for the purpose of private study or research.

- You may not further distribute the material or use it for any profit-making activity or commercial gain

- You may freely distribute the URL identifying the publication in the public portal 


\section{Simulation of ultrafast excited-state dynamics and elastic $x$-ray scattering by quantum wavepacket dynamics}

Cite as: J. Chem. Phys. 151, 104307 (2019); https://doi.org/10.1063/1.5115204

Submitted: 15 June 2019 . Accepted: 18 August 2019 . Published Online: 12 September 2019

Mátyás Pápai (D), Tamás Rozgonyi, Thomas J. Penfold (D), Martin M. Nielsen (D), and Klaus B. Møller (D)

\section{COLLECTIONS}

Paper published as part of the special topic on Ultrafast Spectroscopy and Diffraction from XUV to X-ray Note: This paper is part of the JCP Special Collection on Ultrafast Spectroscopy and Diffraction from XUV to X-ray.
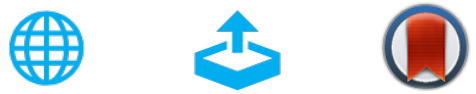

\section{ARTICLES YOU MAY BE INTERESTED IN}

Ultrafast photoelectron spectroscopy of aqueous solutions

The Journal of Chemical Physics 151, 090901 (2019); https://doi.org/10.1063/1.5098402

Excited state dynamics of the s-trans-1, 3-butadiene cation: An ab initio quantum dynamical analysis

The Journal of Chemical Physics 151, 104105 (2019); https://doi.org/10.1063/1.5108610

Electronic and vibrational relaxation dynamics of $\mathrm{NH}_{3}$ Rydberg states probed by vacuumultraviolet time-resolved photoelectron imaging

The Journal of Chemical Physics 151, 104306 (2019); https://doi.org/10.1063/1.5116707

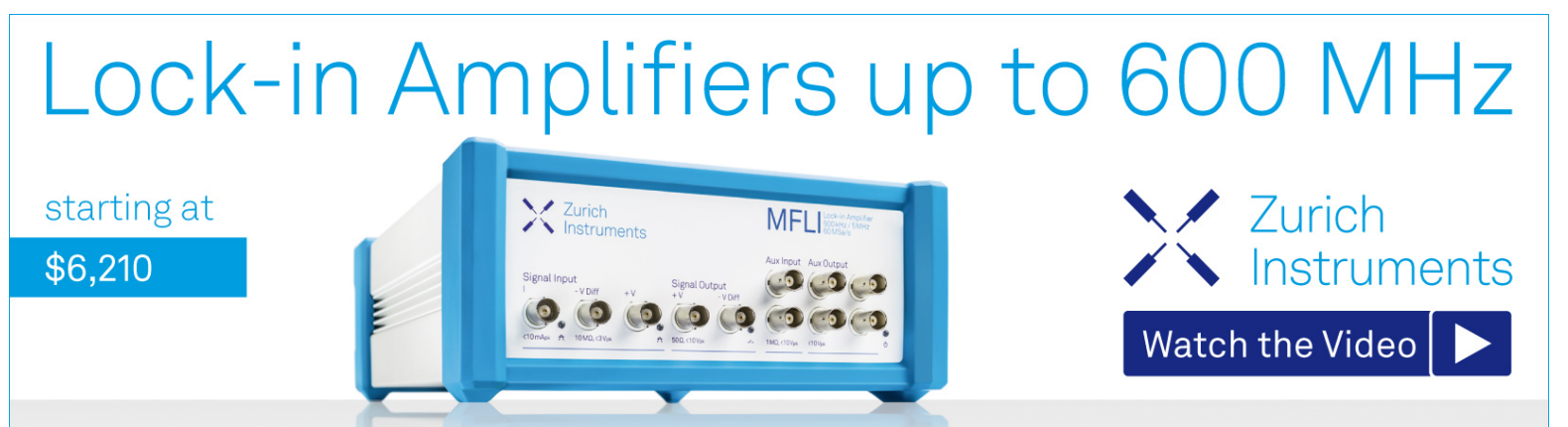




\title{
Simulation of ultrafast excited-state dynamics and elastic $x$-ray scattering by quantum wavepacket dynamics
}

\author{
Cite as: J. Chem. Phys. 151, 104307 (2019); doi: 10.1063/1.51 15204 \\ Submitted: 15 June 2019 • Accepted: 18 August 2019 • \\ Published Online: 12 September 2019
}

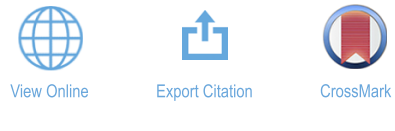

\begin{abstract}
Mátyás Pápai, ${ }^{1, a)}$ (D) Tamás Rozgonyi, ${ }^{2,3}$ Thomas J. Penfold, ${ }^{4}$ (D) Martin M. Nielsen, ${ }^{5}$ (D) and Klaus B. Møller

\section{AFFILIATIONS}

${ }^{1}$ Department of Chemistry, Technical University of Denmark, Kemitorvet 207, DK-2800 Kongens Lyngby, Denmark

${ }^{2}$ Wigner Research Centre for Physics, Hungarian Academy of Sciences, P.O. Box 49, H-1525 Budapest, Hungary

${ }^{3}$ Institute of Materials and Environmental Chemistry, Research Centre for Natural Sciences, Hungarian Academy of Sciences,

P.O. Box 286, H-1519 Budapest, Hungary

${ }^{4}$ Chemistry - School of Natural and Environmental Sciences, Newcastle University, Newcastle upon Tyne

NEl 7RU, United Kingdom

${ }^{5}$ Department of Physics, Technical University of Denmark, Fysikvej 307, DK-2800 Kongens Lyngby, Denmark
\end{abstract}

Note: This paper is part of the JCP Special Collection on Ultrafast Spectroscopy and Diffraction from XUV to X-ray.

a) Electronic mail: papai@kemi.dtu.dk

\begin{abstract}
Simulation of the ultrafast excited-state dynamics and elastic X-ray scattering of the $\left[\mathrm{Fe}(\mathrm{bmip})_{2}\right]^{2+}[\mathrm{bmip}=2$,6-bis(3-methyl-imidazole-1ylidine)-4-pyridine] complex is presented and analyzed. We employ quantum wavepacket dynamics simulations on a 5-dimensional potential energy surface (PES) calculated by time-dependent density functional theory with 26 coupled diabatic states. The simulations are initiated by explicit inclusion of a time-dependent electromagnetic field. In the case of resonant excitation into singlet metal-to-ligand charge transfer $\left({ }^{1}\right.$ MLCT) states, kinetic (exponential) population dynamics are observed with small nuclear motion. In agreement with transient optical absorption spectroscopy experiments, we observe a subpicosecond ${ }^{1}$ MLCT $\rightarrow{ }^{3}$ MLCT intersystem crossing and a subsequent decay into triplet metal-centered $\left({ }^{3} \mathrm{MC}\right)$ states on a picosecond time scale. The simulated time-resolved difference scattering signal is dominated by the ${ }^{3} \mathrm{MC}$ component, for which the structural distortions are significant. On the other hand, excitation into ${ }^{1} \mathrm{MC}$ states leads to ballistic (nonexponential) population dynamics with strong nuclear motion. The reason for these ballistic dynamics is that in this case, the excitation occurs into a nonequilibrium region, i.e., far from the minimum of the ${ }^{1} \mathrm{MC}$ PES. This results in wavepacket dynamics along the principal breathing mode, which is clearly visible in both the population dynamics and difference scattering. Finally, the importance of decomposing the difference scattering into components by electronic states is highlighted, information which is not accessible from elastic X-ray scattering experiments.
\end{abstract}

Published under license by AIP Publishing. https://doi.org/10.1063/1.5115204

\section{INTRODUCTION}

Photoinduced dynamics between electronically excited states are ubiquitous across many fields in physics, chemistry, and even biology. $^{2-4}$ A considerable amount of research effort has been devoted to understand radiationless relaxation processes, i.e., internal conversion (IC) and intersystem crossing (ISC), as well as vibrational dynamics. ${ }^{5-8}$ The ultrashort (femtosecond-picosecond) time scales of these processes have to be experimentally addressed by pump-probe techniques employing very short pulses. In addition to probe pulses with UV, visible, and IR wavelengths, X-ray pulses have been utilized for time-resolved (TR) scattering and spectroscopic experiments. This has recently received a strong impetus from the advent of X-ray Free Electron Lasers ${ }^{9}$ (XFELs) that enable both high brilliance and subpicosecond time resolution. The application of Xray-based techniques is advantageous in many aspects, such as access 
to high-resolution molecular structure ${ }^{10}$ and optically dark states. ${ }^{1}$ However, the recorded data can be extremely complex, leading to difficulties and ambiguities in the interpretation of experimental results.

Theory and computational methods offer powerful complementary tools for the interpretation of X-ray experiments. These can both reduce the complexity and extract additional data that are otherwise hidden, i.e., within a conventional fingerprinting approach. Among various approaches, those are in particular promising that simulate TR spectroscopic or scattering signals, directly enabling assignation and analysis of the experimental data. In the case of spectroscopy, this task requires the combination of electronic structure calculations and nonadiabatic dynamics simulations. This technique has been recently employed for the simulation of TR X-ray absorption $^{12-17}$ and emission spectra. ${ }^{12}$

While X-ray spectroscopies are local, element-specific probes by their nature, X-ray scattering is global, i.e., arising from all electrons in the sample. Henceforth, we utilize the term X-ray diffuse scattering (XDS) in order to differentiate from X-ray diffraction, i.e., scattering on crystals. XDS might arise from several electronic states, as well as solvent molecules, if the measurement is carried out in solution. Therefore, the XDS signal can be especially difficult to interpret; the role of theory is vital here. Debnarova et al. simulated the elastic TR-XDS by the Fourier transform of the quantumchemically calculated electron density. ${ }^{18}$ They found moderate discrepancies from the widely adapted independent atom model (IAM) utilizing the Debye scattering equation, but only for wide angle X-ray scattering (WAXS). Theoretical frameworks for the simulation of TR X-ray scattering of molecules ${ }^{19,20}$ are under continuous development; most recent contributions were achieved by Kirrander et al. ${ }^{21-23}$ Their $a b$ initio method is based on the evaluation of matrix elements of the scattering operator, utilizing high-level multiconfigurational electronic wave functions and nuclear wavepackets (WPs) obtained from quantum dynamics (QD) simulations. Their approach did not only yield high-accuracy results, but also accessed the inelastic and coherent mixed components ${ }^{20,24-26}$ of the scattering signal, in addition to elastic X-ray scattering. However, due to its high computational cost, the method has been applied so far to very small molecules $\left(\mathrm{H}_{2}\right.$ and $\left.\mathrm{CO}_{2}\right)$. In the present work, we study the excited-state dynamics between metal-to-ligand charge transfer (MLCT) and metal-centered (MC) states (Fig. 1) and elastic XDS of an Fe-carbene complex, $\left[\mathrm{Fe}(\mathrm{bmip})_{2}\right]^{2+}[\mathrm{bmip}=2,6$-bis (3-methyl-imidazole-1-ylidine)-4-pyridine]. ${ }^{27,28}$ This molecule and its derivatives have been in the focus of ultrafast spectroscopic ${ }^{27,29-32}$ and scattering ${ }^{33}$ experiments due to their intriguing photophysics, in particular, their long-lived photoactive MLCT states. ${ }^{28}$ We here extend our previously developed model Hamiltonian ${ }^{34}$ to 5 nuclear degrees of freedom (DOF) and implement the electric field of the pump pulse ${ }^{35}$ utilizing different pulse durations and laser frequencies. Subsequently, we convert the time-dependent nuclear wavepacket, evolving in a manifold of electronic states, to a TR elastic difference XDS signal.

This article is organized as follows: Sections II and III review the theory and report the computational details of the QD and XDS simulations, respectively. Section IV presents and analyzes the obtained results for QD (Sec. IV A) and XDS (Sec. IV B). Section V summarizes the most important results, draws the conclusions, and discusses the outlook for future works.
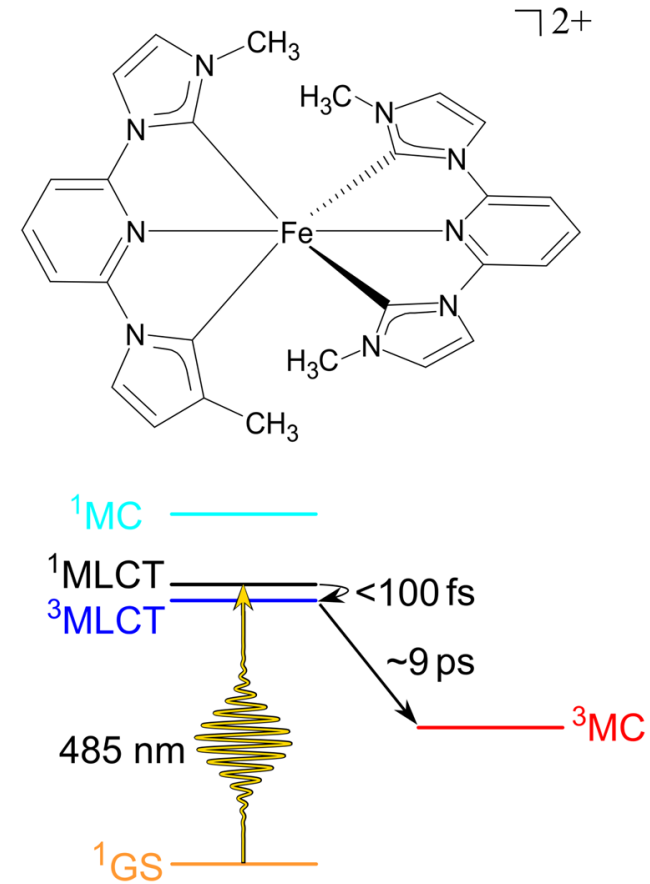

FIG. 1. Molecular structure (top) and MLCT-MC excited-state dynamics of the $\left[\mathrm{Fe}(\mathrm{bmip})_{2}\right]^{2+}$ complex. The lifetimes correspond to the ${ }^{1} \mathrm{MLCT} \rightarrow{ }^{3} \mathrm{MLCT}$ ISC and ${ }^{3} \mathrm{MLCT} \rightarrow{ }^{3} \mathrm{MC}$ decay processes, as obtained from transient optical absorption spectroscopy.

\section{THEORITICAL BACKGROUND}

\section{A. Quantum dynamics}

The ultimate goal of quantum dynamics is to solve the molecular time-dependent Schrödinger equation,

$$
i \hbar \frac{\partial \Psi}{\partial t}=\hat{H} \Psi
$$

where $\Psi$ and $\hat{H}$ are the molecular wave function and Hamiltonian, respectively. In the case of nonadiabatic systems, e.g., the manifold of coupled electronic states of $\left[\mathrm{Fe}(\text { bmip })_{2}\right]^{2+}, \Psi$ can be expanded in the basis of diabatic electronic states $\{|\alpha\rangle\}$,

$$
|\Psi\rangle=\sum_{\alpha=1}^{n_{\mathrm{el}}} \psi^{(\alpha)}(\boldsymbol{R}, t)|\alpha\rangle .
$$

Here, $\psi^{(\alpha)}(\boldsymbol{R}, t)$ is the nuclear wavepacket in the electronic state $\alpha$ and $\boldsymbol{R}$ is the vector of nuclear coordinates. The diabatic electronic basis is convenient to remove the singularities present in the adiabatic representation, by transformation of the derivative nonadiabatic coupling into off-diagonal elements of the electronic Hamiltonian (diabatic coupling). This essentially also leads to smooth potential energy surfaces (PESs) preserving the electronic character.

In the present work, we employ the Multiconfiguration TimeDependent Hartree ${ }^{36-38}$ (MCTDH) method to numerically solve Eq. (1) in multiple dimensions. In MCTDH, within the multiset 
formalism, i.e., Eq. (2), the nuclear wavepacket in each electronic state $|\alpha\rangle$ is expanded in a multiconfigurational series of Hartree products of single particle functions (SPFs),

$$
\begin{aligned}
\psi^{(\alpha)} & \left(R_{1}, \ldots, R_{f}, t\right) \\
& =\sum_{j_{1}^{(\alpha)}=1}^{n_{1}^{(\alpha)}} \ldots \sum_{j_{f}^{(\alpha)}=1}^{n_{f}^{(\alpha)}} A_{j_{1}^{(\alpha)} \ldots j_{f}^{(\alpha)}}(t) \varphi_{j_{1}^{(\alpha)}}^{(1, \alpha)}\left(R_{1}, t\right) \ldots \varphi_{j_{f}^{(\alpha)}}^{(f, \alpha)}\left(R_{f}, t\right),
\end{aligned}
$$

where $A_{j_{1}^{(\alpha)} \ldots j_{f}^{(\alpha)}}(t)$ and $\varphi_{j_{i}^{(\alpha)}}^{(i, \alpha)}\left(R_{i}, t\right)$ are the time-dependent variatonally optimized expansion coefficients and SPFs, respectively, for $f$ nuclear DOF. As seen in Eq. (3), a separate set of SPFs is utilized for each electronic state $|\alpha\rangle$; in this way, the SPFs adapt to the dynamics occurring on each PES. The SPFs are further expanded into a time-independent primitive basis set $\left\{\chi_{k}^{(i)}\left(R_{i}\right)\right\}$,

$$
\varphi_{j_{i}^{(\alpha)}}^{(i, \alpha)}\left(R_{i}, t\right)=\sum_{k=1}^{N_{i}} a_{k j_{i}^{(\alpha)}}^{(i, \alpha)}(t) \chi_{k}^{(i)}\left(R_{i}\right) .
$$

Up to this point, the MCTDH method, as presented, is completely general. Hereby, we choose the nuclear coordinates $\boldsymbol{R}$ as dimensionless mass-frequency weighted coordinates $\boldsymbol{Q}$ of groundstate (GS) normal modes. This is a convenient and widely utilized choice for nonadiabatic dynamics involving relatively small nuclear displacements. The conversion between Cartesian and dimensionless normal mode coordinates is performed by

$$
Q=L\left(x-x_{0}\right),
$$

where $\boldsymbol{x}$ and $\boldsymbol{x}_{\mathbf{0}}$ are the actual and reference, i.e., Franck-Condon (FC) geometries in Cartesian coordinates, respectively. The $\boldsymbol{L}$ transformation matrix contains the eigenvectors of the mass-weighted Hessian, scaled by $\sqrt{m_{a} \Omega_{i} / \hbar}$, where $m$ and $\Omega$ are the masses and vibrational frequencies, for each atom $a$ and normal mode $i$. Consistent with normal modes, we specify the primitive basis $\left\{\chi_{k}^{(i)}\left(Q_{i}\right)\right\}$ to be a Hermite discrete variable representation (DVR), ${ }^{3}$ i.e., a grid representation of one-dimensional harmonic oscillator eigenfunctions.

\section{B. Spin-vibronic and interaction Hamiltonians}

The molecular Hamiltonian utilized for the QD simulations of this work is expressed as

$$
\boldsymbol{H}(t)=\left(\hat{T}_{N}+V_{0}\right) \mathbf{1}+\boldsymbol{W}+\mathcal{S}+\boldsymbol{\mu \varepsilon} E(t) .
$$

With the exception of the last term, the right-hand side of Eq. (6) defines the diabatic spin-vibronic Hamiltonian. The first two terms are the kinetic and potential energy operators of the zerothorder Hamiltonian for the electronic ground state (GS) within the harmonic oscillator approximation, respectively,

$$
\hat{H}_{0}=\hat{T}_{N}+V_{0}=\sum_{i=1}^{f} \frac{\Omega_{i}}{2}\left(\frac{\partial^{2}}{\partial Q_{i}^{2}}+Q_{i}^{2}\right) .
$$

In Eq. (6), $\mathbf{1}$ is the unity matrix of dimension $n_{\mathrm{el}}$. The $\boldsymbol{W}$ matrix expresses the difference between ground-state and excited-state PESs and the diabatic coupling between excited states. As mentioned, diabatic PESs are smooth by their nature; this allows us to expand $\boldsymbol{W}$ in a Taylor series, in the present case up to second order, around the FC geometry,

$$
W=W^{(0)}+W^{(1)}+W^{(2)} .
$$

$W^{(0)}$ is a $\mathbf{Q}$-independent diagonal matrix, containing the vertical excitation energies from the GS to the excited states at the FC geometry. $W^{(\mathbf{1})}$ has both on- and off-diagonal elements, which depend linearly on $\boldsymbol{Q}$. The on-diagonal terms express the forces acting on the excited-state PESs, relative to the forces on the groundstate PES. The off-diagonal counterparts are the linear approximation of the nonadiabatic couplings. $\boldsymbol{W}^{(2)}$ is a $\boldsymbol{Q}^{2}$-dependent diagonal matrix, whose elements describe the changes between ground- and excited-state vibrational frequencies.

The above-discussed first three terms of the Hamiltonian of Eq. (6) define the widely used vibronic-coupling Hamiltonian. ${ }^{39-41}$ As the excited-state dynamics of $\left[\mathrm{Fe}(\mathrm{bmip})_{2}\right]^{2+}$ involve both singlet and triplet electronic states, ${ }^{34}$ extension of the Hamiltonian by the inclusion of spin-orbit coupling (SOC) is necessary; this leads to the spin-vibronic Hamiltonian. ${ }^{7,34,42-46} \mathcal{S}$ in Eq. (6) is the SOC matrix, whose off-diagonal elements describe the singlet-triplet and triplet-triplet SOC.

The last component of our Hamiltonian in Eq. (6) is an interaction term between the transition dipole moment (TDM) $\boldsymbol{\mu}$ of $\left[\mathrm{Fe}(\mathrm{bmip})_{2}\right]^{2+}$ and the electric field of the linearly polarized pump pulse that promotes the molecule to an electronic excited state, from the ground state. The polarization vector $\varepsilon$ is the vectorial component of the field and $E(t)$ is the time-dependent amplitude with a Gaussian profile,

$$
E(t)=E_{0} e^{-2 \ln (2)\left(t-t_{0}\right)^{2} / \tau^{2}} \cos \left(\omega\left(t-t_{0}\right)\right),
$$

where $E_{0}$ is the field amplitude, $\tau$ is the intensity full width at half maximum (FWHM), $\omega$ is the angular frequency of the pump pulse, and $t_{0}$ is the time shift of the center of the pulse.

$\left[\mathrm{Fe}(\text { bmip })_{2}\right]^{2+}$ has $\mathrm{D}_{2 \mathrm{~d}}$ molecular symmetry in the electronic ground state. As such, excitation into electronically degenerate states can occur; this will be in fact the case in the present work, as 2-fold degenerate states, i.e., belonging to the E irreducible representation of the $\mathrm{D}_{2 \mathrm{~d}}$ point group, will be excited (see Sec. IV A). In order to avoid artificial interference of relaxation pathways, ${ }^{35}$ we set $\varepsilon$ to be parallel to the TDM associated with an electronic transition from the ground state to one of the two components of the pumped degenerate manifold.

\section{Elastic x-ray scattering}

First, we review the theory of elastic X-ray scattering in a single electronic state, and we thus drop the electronic state index $\alpha$. Scattering signals are cast in terms of the scattering vector $\boldsymbol{q}$,

$$
q=k_{\text {in }}-k_{\text {sc }},
$$

where $\boldsymbol{k}_{\mathrm{in}}$ and $\boldsymbol{k}_{\mathrm{sc}}$ are the wave vectors of the incident and scattered $\mathrm{X}$-ray photons, respectively. $\boldsymbol{q}$ is commonly expressed in units of $\AA^{-1}$. In the case of elastic XDS, $\left|\boldsymbol{k}_{\text {in }}\right|=\left|\boldsymbol{k}_{\mathrm{sc}}\right|=k$ and 


$$
|\boldsymbol{q}|=q=2 k \sin (\Theta / 2),
$$

where $\Theta$ is the scattering angle between $\boldsymbol{k}_{\text {in }}$ and $\boldsymbol{k}_{\text {sc }}$.

The scattering signal $S(\boldsymbol{q})$ in units of the Thomson scattering cross section (electronic units, e.u.) can, under a reasonable set of approximations, ${ }^{47}$ e.g., neglecting electronic coherence, be written as

$$
S(\boldsymbol{q})=\left\langle\psi(\boldsymbol{R}) \|\left. F(\boldsymbol{R}, \boldsymbol{q})\right|^{2} \mid \psi(\boldsymbol{R})\right\rangle=\int \rho_{\mathrm{N}}(\boldsymbol{R})|F(\boldsymbol{R}, \boldsymbol{q})|^{2} d \boldsymbol{R},
$$

where $\rho_{\mathrm{N}}$ is the nuclear density. The central quantity in Eq. (12) and elastic XDS is the molecular form factor $F(\boldsymbol{R}, \boldsymbol{q})$,

$$
F(\boldsymbol{R}, \boldsymbol{q})=\left\langle\phi(\boldsymbol{r} ; \boldsymbol{R})\left|e^{i \boldsymbol{q} \cdot \boldsymbol{r}}\right| \phi(\boldsymbol{r} ; \boldsymbol{R})\right\rangle=\int \rho_{\mathrm{e}}(\boldsymbol{r} ; \boldsymbol{R}) e^{i \boldsymbol{q} \cdot \boldsymbol{r}} d \boldsymbol{r} .
$$

Here, $\boldsymbol{r}$ denotes the vector of electronic coordinates; $\phi(\boldsymbol{r} ; \boldsymbol{R})$ and $\rho(\boldsymbol{r} ; \boldsymbol{R})$ are the electronic wave function and density, respectively. Equation (13) leads to the well-known fact that the molecular form factor gives the Fourier transform of the electron density.

We now employ the independent atom model, in which scattering from independent atoms with isolated, perfectly spherical electron densities is assumed. This leads to the approximation of the molecular form factor by the sum of atomic form factors

$$
F(\boldsymbol{R}, \boldsymbol{q}) \sim \sum_{i=1}^{n_{\mathrm{at}}} f_{i}(q) e^{i \boldsymbol{q} \cdot \boldsymbol{R}_{i}}
$$

with the atomic form factors written as a linear combination of Gaussians

$$
f(q)=\sum_{m=1}^{4} a_{m} e^{-b_{m}(q / 4 \pi)^{2}}+c,
$$

where $a_{i}, b_{i}$, and $\mathrm{c}$ are the tabulated Cromer-Mann coefficients. In the present work, we simulate the isotropic scattering signal, which implies that the probability of finding the molecule in any orientation is taken to be identical. Although XDS obtained by ultrafast pump-probe experiments contains an anisotropic component as well, decomposition of the experimental signal into isotropic and anisotropic parts allows comparison to the signal simulated by the present approach. ${ }^{47,48}$ Utilizing nuclear pair densities, the isotropic scattering signal is written as ${ }^{49}$

$$
S(q)=\sum_{i} f_{i}^{2}(q)+\sum_{i} \sum_{j \neq i} f_{i}(q) f_{j}(q) 4 \pi \int_{0}^{\mathcal{R}} \rho_{i j}(D) \frac{\sin (q D)}{q D} D^{2} d D,
$$

where $\rho_{i j}(D)$ is the nuclear pair density, $D$ is the distance between atoms $i$ and $j$, and $\mathcal{R}$ is the radius of the sphere representing the coherence volume, over which the X-ray beam is coherent. Equation (16) can be used to derive $S(q)$ in terms of radial distribution functions, ${ }^{49}$ readily obtainable from molecular dynamics (MD) simulations. In the present work, we utilize the time-dependent center of the nuclear wavepacket in each electronic state $|\alpha\rangle$,

$$
\left\langle Q_{i}^{(\alpha)}\right\rangle(t)=\frac{\left\langle\psi^{(\alpha)}(t)\left|Q_{i}\right| \psi^{(\alpha)}(t)\right\rangle}{\left\langle\psi^{(\alpha)}(t) \mid \psi^{(\alpha)}(t)\right\rangle}=\frac{\left\langle\psi^{(\alpha)}(t)\left|Q_{i}\right| \psi^{(\alpha)}(t)\right\rangle}{p^{(\alpha)}(t)},
$$

to calculate the scattering signal. In Eq. $(17), p^{(\alpha)}(t)$ are the timedependent electronic populations, whose sum is normalized to unity. Therefore, the nuclear distributions shrink into delta functions centered at the wavepacket centroids, leading to the Debye scattering equation,

$$
S(q, t)=\sum_{i} \sum_{j} f_{i}(q) f_{j}(q) \frac{\sin \left(q D_{i j}(t)\right)}{q D_{i j}(t)} .
$$

We point out that $\left\langle Q_{i}^{(\alpha)}\right\rangle(t)$ corresponds to the often-used quantity to fit experimental elastic XDS signals. Some of us employed this approach in a recent XDS work on a complex related to $\left[\mathrm{Fe}(\mathrm{bmip})_{2}\right]^{2+},{ }^{10}$ where, in that case, we utilized density functional theory (DFT)-optimized and distorted structures, to fit the experimental signal, which, in turn, resulted in time-dependent structures. This approach utilizing solely the wavepacket centroids neglects the wavepacket width; we address and discuss this issue in Sec. IV B.

Note the difference in nuclear coordinates between Eqs. (17) and (18): while the former utilizes dimensionless normal coordinates, the latter is cast in terms of interatomic distances (in units of $\AA$ ). We connect these two equations by the transformation between normal and Cartesian coordinates; see Eq. (5).

Equation (18) is applied for the Cartesian geometries resulting from $\left\langle Q_{i}^{(\alpha)}\right\rangle(t)$, for each electronic state $|\alpha\rangle$, at each time $t$ of the QD simulation, leading to $S^{(\alpha)}(q, t)$. The simulated time-dependent scattering signal is then obtained by the sum of $S^{(\alpha)}(q, t)$, weighted by the electronic populations

$$
S(q, t)=\sum_{\alpha=1}^{n_{\mathrm{el}}} p^{(\alpha)}(t) S^{(\alpha)}(q, t) .
$$

Finally, the differential scattering signal $\Delta S$ is calculated by taking the difference of the pumped and the nonpumped signal,

$$
\Delta S(q, t)=S(q, t)-S(q, 0) .
$$

We close this section by analyzing the validity of the presented elastic XDS methodology. The IAM leads to the efficient calculation of the XDS signal via the Debye scattering equation [Eq. (18)]. However, the IAM is a rather simplistic model that importantly neglects chemical bonding and does not distinguish between electronic states. This has recently been confirmed by comparison to $a b$ initio-derived scattering signals, and the largest deviations were seen for very small molecules, such as $\mathrm{H}_{2}{ }^{22}$ This is because the electron density of these systems is dominated by valence electrons, for which the IAM fails. On the other hand, for larger molecules, the IAM performs significantly better. For instance, in the case of stilbene, the IAM differential elastic XDS signal is basically identical to the one derived by $a b$ initio calculations up to $q=2 \AA^{-1}$; even at larger $q$ values, the main features are well reproduced, although the IAM overestimates the difference signal. ${ }^{18}$ For transition metal complexes, as is the $\left[\mathrm{Fe}(\mathrm{bmip})_{2}\right]^{2+}$ molecule studied in the present work, the situation is even more favorable due to their high number of core electrons. Furthermore, for photophysics, such as IC and ISC without any bond cleavage, the IAM is expected to perform well for the simulation of difference elastic scattering signals. It is also important to point out that although the Debye scattering equation in itself reflects only the nuclear structure, not the electronic state, 
we utilize Eq. (18) for each state $|\alpha\rangle$. Thus, the simulated $\Delta S(q, t)$ does reflect the dynamics in each electronic state, through the electronic populations $p^{(\alpha)}$. Finally, we recall that we employ Eq. (18) only for the wavepacket centroids without accounting for the width; we will come back to this point in Sec. IV B.

\section{METHODOLOGICAL DETAILS}

\section{A. Quantum dynamics simulations}

The QD simulations were carried out using the Heidelberg MCTDH code. ${ }^{37}$ The initial nuclear wavepacket was built from onedimensional ground-state vibrational harmonic oscillator eigenfunctions on the ground-state PES, as obtained from propagation in imaginary time (relaxation). The electronic excitation is achieved by the interaction Hamiltonian of Eq. (6); further details are described in Sec. III B. In Table II (above Appendix A), we present the computational details of the simulations, i.e., the size of SPFs and primitive basis sets. These parameters ensured convergence for the whole 4 ps duration of the simulations.

\section{B. The Hamiltonian}

The Hamiltonian used for the QD simulations in this work is based on our previous studies. ${ }^{34,35}$ It includes 5 normal modes, and 5 singlet and 7 triplet electronic states, accounting for the 3 $(-1,0,1)$ spin components of the triplet states, 26 states, overall. The original spin-vibronic Hamiltonian of Refs. 34 and 35 contained 4 nuclear DOF. These are all Fe-N/C stretching modes, accounting for the most important nuclear (vibrational) motion in the excited states (tuning modes, $v_{6}$ and $v_{36}$ ) and nonadiabatic coupling between excited states (coupling modes, $v_{11}$ and $v_{25}$ ). In addition to these 4 modes, in the present work, we add another tuning mode, $v_{12}$, the symmetric counterpart of $v_{11}$ that has a predominantly out-of-phase $\mathrm{Fe}-\mathrm{N} / \mathrm{C}$ stretching character. We recently identified $v_{12}$ as a potential tuning mode and hereby investigate how its inclusion affects the simulated excited-state dynamics.

The electronic states of our model are the following: the electronic ground state $\left({ }^{1} \mathrm{GS}\right)$, a pair of degenerate singlet MLCT $\left({ }^{1} \mathrm{MLCT}\right)$ and $\mathrm{MC}\left({ }^{1} \mathrm{MC}\right), 4$ triplet MLCT $\left({ }^{3} \mathrm{MLCT}\right)$, and 3 triplet MC $\left({ }^{3} \mathrm{MC}\right)$ states. The excited states were obtained using timedependent density functional theory (TD-DFT) within the TammDancoff approximation $^{50}$ (TDA), at the TD-B3LYP ${ }^{* 51} /$ TZVP $^{52}$ level of theory utilizing the ORCA3.0 program package. ${ }^{53}$ The resolution of identity ${ }^{54}$ and chain of spheres ${ }^{55}$ (RIJCOSX) methods were utilized to approximate the two electron integrals. We recently have shown that the excited-state PESs of $\left[\mathrm{Fe}(\text { bmip })_{2}\right]^{2+}$ are not influenced by a polar solvent, such as acetonitrile; ${ }^{56}$ we thus carried out all TD-DFT calculations in vacuum. As these calculations lead to a set of adiabatic states, a diabatization procedure is needed to transform into a diabatic electronic basis required for QD simulations. We employ the procedure of diabatization by ansatz. ${ }^{7,57}$ In this method, a diabatic to adiabatic transformation by diagonalization is exploited to optimize the coefficients of the vibronic-coupling Hamiltonian (VCHAM) to the adiabatic PESs, calculated by quantum chemistry. The fit is performed separately for singlet and triplet states utilizing the VCHAM utility of the Heidelberg MCTDH program package. ${ }^{37}$ The SOC matrix $\mathcal{S}$ is calculated at the FC geometry by a perturbative approach ${ }^{58}$ within the zeroth-order regular approximation (ZORA). ${ }^{59}$ These calculations were carried out at the $\mathrm{B} \mathrm{LYP}^{*} / \mathrm{TZP}$ level of theory using the ADF2012.01 software. ${ }^{60} \mathrm{We}$ ensured the consistency of ORCA and ADF-calculated electronic states.

The diabatic excited-state PESs along the dominant breathing mode for the dynamics, $v_{6}$, and the newly added mode $v_{12}$ are shown in Fig. 2. We present the electronic energies, oscillator strengths, and dominant electronic configurations at the FC geometry in Table I. In Fig. 3, we illustrate the MLCT and MC characters by natural transition orbitals (NTOs) of the singlet excited states. We note that all excited states can be described by one dominant electron configuration/NTO pair, with the exception of $\mathrm{T}_{4}$ and $\mathrm{T}_{7}$, for which two configurations have equal weights (see Table I). Furthermore, NTOs occurring for the same hole/particle, for different excited states (including the triplets), are very similar.
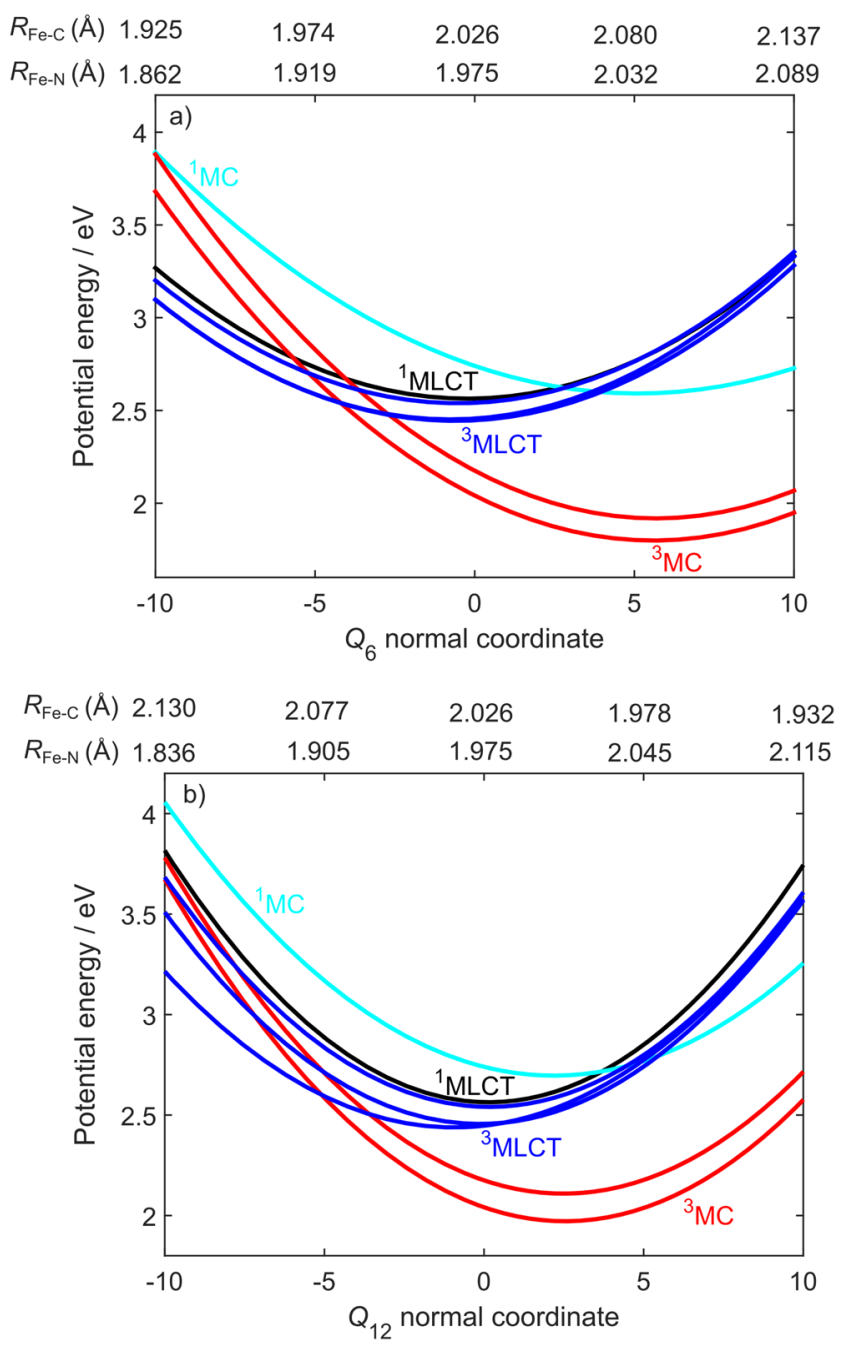

FIG. 2. Diabatic excited-state PESs along (a) $v_{6}$ and (b) $v_{12}$. $Q_{6}$ and $Q_{12}$ are dimensionless normal coordinates. For comparison, the corresponding $\mathrm{Fe}-\mathrm{N}$ and $\mathrm{Fe}-\mathrm{C}$ bond lengths are also shown. 
TABLE I. Characters (symmetries within the $D_{2 d}$ point group), energies $(E)$, oscillator strengths $(f)$, and dominant electronic configurations of the electronic states $|\alpha\rangle$, as obtained by TD-B3LYP*/TZVP calculations. For the illustration of electronic configurations, see the NTOs shown in Fig. 3.

\begin{tabular}{llccc}
\hline \hline$|\alpha\rangle$ & Character & $E(\mathrm{eV})$ & $f$ & Electronic configuration \\
\hline $\mathrm{S}_{0}$ & ${ }^{1} \mathrm{GS}\left({ }^{1} \mathrm{~A}_{1}\right)$ & 0.000 & $\ldots$ & $3 d_{x z}^{2} 3 d_{y z}^{2} 3 d_{x y}^{2} \mathrm{~L}_{1}^{0}-\pi^{*} \mathrm{~L}_{2}^{0}-\pi^{*} 3 d_{z^{2}}^{0}$ \\
\hline $\mathrm{S}_{1}$ & ${ }^{1} \mathrm{MLCT}\left({ }^{1} \mathrm{E}\right)$ & 2.564 & 0.0017 & $3 d_{x z}^{2} 3 d_{y z}^{2} 3 d_{x y}^{1} \mathrm{~L}_{1}^{1}-\pi^{*} \mathrm{~L}_{2}^{0}-\pi^{*} 3 d_{z^{2}}^{0}$ \\
$\mathrm{~S}_{2}$ & ${ }^{1} \mathrm{MLCT}\left({ }^{1} \mathrm{E}\right)$ & 2.564 & 0.0017 & $3 d_{x z}^{2} 3 d_{y z}^{2} 3 d_{x y}^{1} \mathrm{~L}_{1}^{0}-\pi^{*} \mathrm{~L}_{2}^{1}-\pi^{*} 3 d_{z^{2}}^{0}$ \\
\hline $\mathrm{S}_{3}$ & ${ }^{1} \mathrm{MC}\left({ }^{1} \mathrm{E}\right)$ & 2.740 & 0.0002 & $3 d_{x z}^{1} 3 d_{y z}^{2} 3 d_{x y}^{2} \mathrm{~L}_{1}^{0}-\pi^{*} \mathrm{~L}_{2}^{0}-\pi^{*} 3 d_{z^{2}}^{1}$ \\
$\mathrm{~S}_{4}$ & ${ }^{1} \mathrm{MC}\left({ }^{1} \mathrm{E}\right)$ & 2.740 & 0.0002 & $3 d_{x z}^{2} 3 d_{y z}^{1} 3 d_{x y}^{2} \mathrm{~L}_{1}^{0}-\pi^{*} \mathrm{~L}_{2}^{0}-\pi^{*} 3 d_{z^{2}}^{1}$ \\
\hline $\mathrm{T}_{1}$ & ${ }^{3} \mathrm{MC}\left({ }^{3} \mathrm{E}\right)$ & 2.041 & 0.0000 & $3 d_{x z}^{1} 3 d_{y z}^{2} 3 d_{x y}^{2} \mathrm{~L}_{1}^{0}-\pi^{*} \mathrm{~L}_{2}^{0}-\pi^{*} 3 d_{z^{2}}^{1}$ \\
$\mathrm{~T}_{2}$ & ${ }^{3} \mathrm{MC}\left({ }^{3} \mathrm{E}\right)$ & 2.041 & 0.0000 & $3 d_{x z}^{2} 3 d_{y z}^{1} 3 d_{x y}^{2} \mathrm{~L}_{1}^{0}-\pi^{*} \mathrm{~L}_{2}^{0}-\pi^{*} 3 d_{z^{2}}^{1}$ \\
$\mathrm{~T}_{3}$ & ${ }^{3} \mathrm{MC}\left({ }^{3} \mathrm{~B}_{1}\right)$ & 2.175 & 0.0000 & $3 d_{x z}^{2} 3 d_{y z}^{2} 3 d_{x y}^{1} \mathrm{~L}_{1}^{0}-\pi^{*} \mathrm{~L}_{2}^{0}-\pi^{*} 3 d_{z^{2}}^{1}$ \\
\hline $\mathrm{T}_{4}$ & ${ }^{3} \mathrm{MLCT}\left({ }^{3} \mathrm{~A}_{1}\right)$ & 2.447 & 0.0000 & $3 d_{x z}^{1} 3 d_{y z}^{2} 3 d_{x y}^{2} \mathrm{~L}_{1}^{0}-\pi^{*} \mathrm{~L}_{2}^{1}-\pi^{*} 3 d_{z^{2}}^{0}$ \\
& & & & $3 d_{x z}^{2} 3 d_{y z}^{1} 3 d_{x y}^{2} \mathrm{~L}_{1}^{1}-\pi^{*} \mathrm{~L}_{2}^{0}-\pi^{*} 3 d_{z^{2}}^{0}$ \\
$\mathrm{~T}_{5}$ & ${ }^{3} \mathrm{MLCT}\left({ }^{3} \mathrm{E}\right)$ & 2.456 & 0.0000 & $3 d_{x z}^{2} 3 d_{y z}^{2} 3 d_{x y}^{1} \mathrm{~L}_{1}^{1}-\pi^{*} \mathrm{~L}_{2}^{0}-\pi^{*} 3 d_{z^{2}}^{0}$ \\
$\mathrm{~T}_{6}$ & ${ }^{3} \mathrm{MLCT}\left({ }^{3} \mathrm{E}\right)$ & 2.456 & 0.0000 & $3 d_{x z}^{2} 3 d_{y z}^{2} 3 d_{x y}^{1} \mathrm{~L}_{1}^{0}-\pi^{*} \mathrm{~L}_{2}^{1}-\pi^{*} 3 d_{z^{2}}^{0}$ \\
$\mathrm{~T}_{7}$ & ${ }^{3} \mathrm{MLCT}\left({ }^{3} \mathrm{~B}_{2}\right)$ & 2.541 & 0.0000 & $3 d_{x z}^{1} 3 d_{y z}^{2} 3 d_{x y}^{2} \mathrm{~L}_{1}^{0}-\pi^{*} \mathrm{~L}_{2}^{1}-\pi^{*} 3 d_{z^{2}}^{0}$ \\
& & & & $3 d_{x z}^{2} 3 d_{y z}^{1} 3 d_{x y}^{2} \mathrm{~L}_{1}^{1}-\pi^{*} \mathrm{~L}_{2}^{0}-\pi^{*} 3 d_{z^{2}}^{0}$ \\
\hline \hline
\end{tabular}

For the interaction Hamiltonian of Eq. (6), the TDM vectors to the ${ }^{1}$ MLCT and ${ }^{1} \mathrm{MC}$ are taken from Ref. 35, also calculated at the TD-B3LYP $/$ TZVP level. We utilize two values for both pulse parameters $\tau$ and $\omega . \tau=10 \mathrm{fs}\left(t_{0}=20 \mathrm{fs}\right)$ approaches the limit of
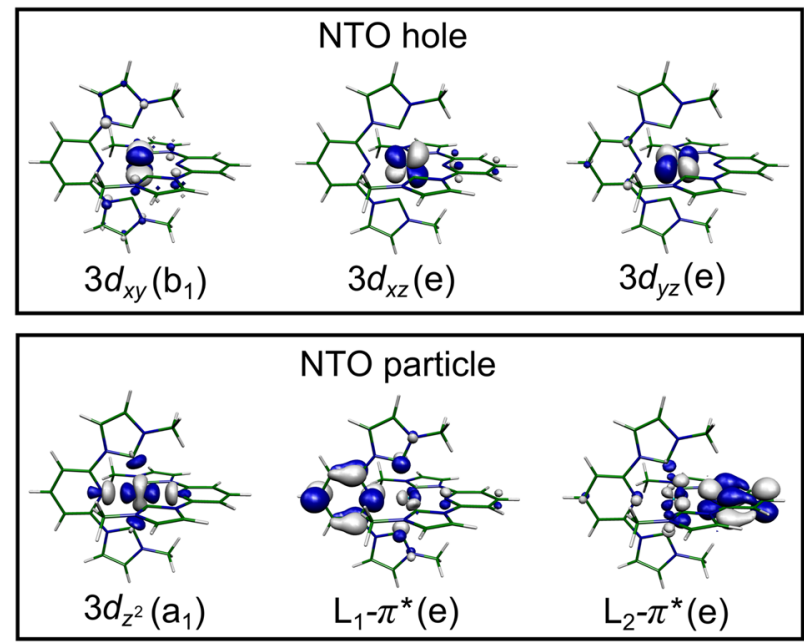

FIG. 3. TD-DFT NTOs for the characterization of MLCT and MC states (plotted for the ${ }^{1} \mathrm{MLCT}$ and ${ }^{1} \mathrm{MC}$ states). The labels in parantheses are orbital symmetries within the $D_{2 d}$ point group. These NTOs illustrate the dominant electronic configurations shown in Table I. instantaneous (impulsive) excitation, while $\tau=60 \mathrm{fs}$ ( $t_{0}=100 \mathrm{fs}$ ) reflects a more realistic pump pulse duration for ultrafast experiments (e.g., the transient absorption measurements of $\left[\mathrm{Fe}(\mathrm{bmip})_{2}\right]^{2+}$ in Ref. 27). We set the field angular frequency $\omega$ to be resonant with the ${ }^{1} \mathrm{GS} \rightarrow{ }^{1} \mathrm{MLCT}$ electronic excitation, and $0.2 \mathrm{eV}$ above this resonance energy (off-resonant with the ${ }^{1}$ MLCT), with the aim of exciting the ${ }^{1} \mathrm{MC}$ states. For the field amplitude, we apply $E_{0}=0.0054$ a.u.; this value ensures that no Rabi oscillations occur between the ground and excited states.

The parameters of the applied spin-vibronic Hamiltonian for the original four modes $\left(v_{6}, v_{11}, v_{25}\right.$, and $\left.v_{36}\right)$ are taken from Ref. 34 . We report the corresponding coefficients of the Hamiltonian and fits to adiabatic PESs for the new mode $v_{12}$ in Appendix A.

\section{Elastic x-ray scattering}

We implemented Eqs. (18)-(20) in MATLAB2019a ${ }^{61}$ for simulating the time-resolved elastic XDS signal. For this, we extracted $\left\langle Q_{i}^{(\alpha)}\right\rangle(t)$ and $p^{(\alpha)}(t)$ from each 2 fs of the QD simulation. The Cromer-Mann coefficients were taken from Ref. 62. In the case of iron, values for $\mathrm{Fe}^{2+}$ were used.

\section{RESULTS AND DISCUSSION}

\section{A. Quantum dynamics}

The results of the four QD simulations utilizing different excitation conditions are shown in Fig. 4. First, we discuss the case of the excitation resonant to the ${ }^{1}$ MLCT states. In Figs. 4(a) and 4(b), 

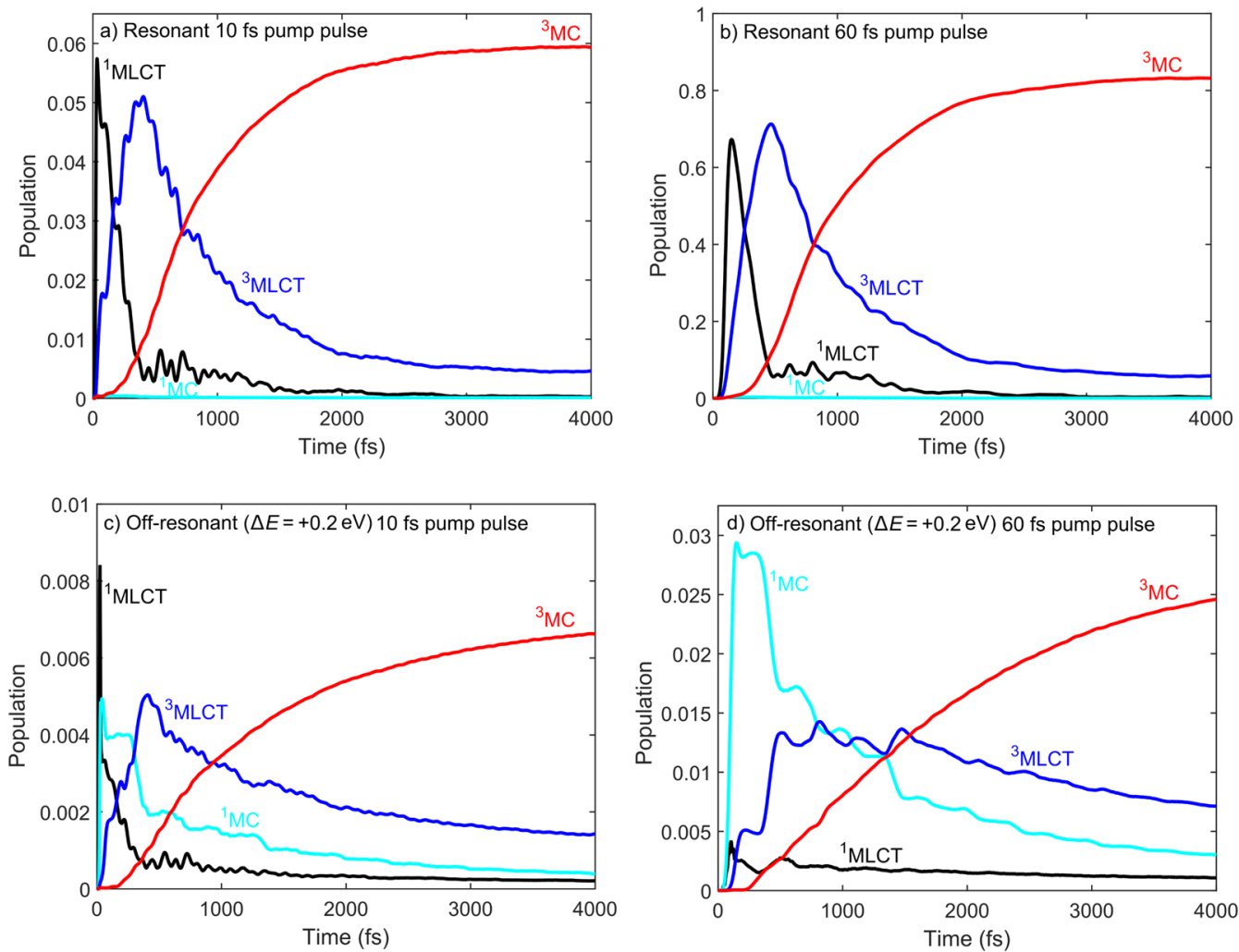

FIG. 4. Excited-state population dynamics from the QD simulations. The four panels correspond to different excitation conditions: (a) $\tau=10 \mathrm{fs}\left(t_{0}=20 \mathrm{fs}\right), \omega$ resonant to the ${ }^{1} \mathrm{GS} \rightarrow{ }^{1} \mathrm{MLCT}$ excitation, (b) $\tau=60 \mathrm{fs}\left(t_{0}=100 \mathrm{fs}\right.$ ), $\omega$ resonant to the ${ }^{1} \mathrm{GS} \rightarrow{ }^{1} \mathrm{MLCT}$ excitation, (c) $\tau=10 \mathrm{fs}\left(t_{0}=20 \mathrm{fs}\right), \omega-0.2 \mathrm{eV}$ above ${ }^{1} \mathrm{MLCT}$ resonance, and (d) $\tau=60 \mathrm{fs}\left(t_{0}=100 \mathrm{fs}\right), \omega-0.2 \mathrm{eV}$ above ${ }^{1} \mathrm{MLCT}$ resonance.

we present the population dynamics using $10 \mathrm{fs}$ and $60 \mathrm{fs}$ pump pulses, respectively. In both cases, the initial ${ }^{1} \mathrm{GS} \rightarrow{ }^{1}$ MLCT excitation is followed by a $\sim 100$ fs ISC to ${ }^{3}$ MLCT states, which decay into ${ }^{3} \mathrm{MCs}$ on a picosecond time scale. These dynamics follow exponential kinetics, which we attribute to the weak nuclear motion in the MLCT states, as the wavepacket is excited to an equilibrium region, i.e., close to the minimum of the ${ }^{1}$ MLCT PES. In particular, the ${ }^{3} \mathrm{MLCT} /{ }^{3} \mathrm{MC}$ intersection is not reached, and the wavepacket thus slowly leaks from ${ }^{3} \mathrm{MLCT}$ into the ${ }^{3} \mathrm{MC}$ states. These results are in good agreement with our previous QD simulations using the $4 \mathrm{D}$ Hamiltonian with impulsive excitation, ${ }^{34}$ as well as with experimental observations. ${ }^{27}$ However, in comparison to Refs. 34 and 35 , the ${ }^{3} \mathrm{MC}$ rise faster during the first picosecond, due to the inclusion of the mode $v_{12}$. Furthermore, as is clear from Figs. 4(a) and 4 (b), the duration of the pump pulse does not influence the excited-state dynamics. The only notable difference is the damping of the fast ${ }^{1}$ MLCT $\leftrightarrow{ }^{3}$ MLCT oscillations for the $60 \mathrm{fs}$ pulse. This is expected, ${ }^{8,63}$ as the pulse duration approaches the period of the observed oscillations. These results also justify the utilization of impulsive excitation to the ${ }^{1}$ MLCT states for the simulation of excited-state dynamics of $\left[\mathrm{Fe}(\mathrm{bmip})_{2}\right]^{2+}$.

We now investigate the effect of tuning the laser frequency, $\omega$, $0.2 \mathrm{eV}$ above the ${ }^{1}$ MLCT resonance. Figures 4(c) and 4(d) present the results for $10 \mathrm{fs}$ and $60 \mathrm{fs}$ pump pulses, respectively. In the $10 \mathrm{fs}$ case, both ${ }^{1}$ MLCT and ${ }^{1} \mathrm{MC}$ states are excited with a $\sim 2: 1$ ratio. This is because although $\omega$ is now off resonant with the ${ }^{1}$ MLCT and resonant with the ${ }^{1} \mathrm{MC}$ states, the $10 \mathrm{fs}$ pulse has a large spectral width that allows excitation into both ${ }^{1}$ MLCT and ${ }^{1} \mathrm{MC}$ states. Note also that the oscillator strengths to ${ }^{1}$ MLCT states are one order of magnitude larger than those to the ${ }^{1} \mathrm{MCs}$ (see Table I). Excitation into the ${ }^{1} \mathrm{MC}$ state leads to the appearance of oscillations with a period of $330 \mathrm{fs}$, most apparent for the ${ }^{1} \mathrm{MC}$ population. These oscillations are assigned to wavepacket dynamics along the breathing mode $v_{6}$, whose period matches the one of mode $v_{6}$. The reason for this oscillatory signal is the creation of the ${ }^{1} \mathrm{MC}$ wavepacket in a nonequilibrium region, i.e., displaced from the minimum of the ${ }^{1} \mathrm{MC}$ PES; this displacement is the largest along $v_{6}$. Therefore, vibrational dynamics takes place in the ${ }^{1} \mathrm{MC}$ along $v_{6}$, which modulates the population transfer between ${ }^{1} \mathrm{MC}$ and MLCT states. The dynamics between the ${ }^{1} \mathrm{MC}$ and ${ }^{1,3} \mathrm{MLCT}$ states are thus not kinetic, but ballistic. This is a result of the fact that due to the nuclear motion in the ${ }^{1} \mathrm{MC}$ states, the wavepacket reaches the ${ }^{1} \mathrm{MC} /{ }^{1,3} \mathrm{MLCT}$ crossing, where a transition into the MLCT states takes place; this is reflected in the abrupt steps of the ${ }^{1} \mathrm{MC}$ population in Fig. 4(c). We here mention that a ballistic mechanism has recently been proposed for photoinduced ISCs. $^{64,65}$ 
In the following, we discuss the dynamics resulting from excitation by the 60 fs pump pulse; these results are shown in Fig. 4(d). As seen in the figure, only a small fraction of the population is excited to the ${ }^{1}$ MLCT states; the dominant excitation process is now ${ }^{1} \mathrm{GS} \rightarrow{ }^{1} \mathrm{MC}$. This is due to the fact that the $60 \mathrm{fs}$ pulse has a narrow spectral width, and as $\omega$ is chosen to be $0.2 \mathrm{eV}$ off-resonant to the ${ }^{1}$ MLCT states, the excitation fraction for these states significantly decreases. The ballistic wavepacket dynamics is now more apparent, also for the MLCT states, than for the $10 \mathrm{fs}$ pump pulse, which is attributed to the selective excitation into the ${ }^{1} \mathrm{MC}$ states. These results confirm the activation and dominance of $v_{6}$ for the excited-state dynamics and highlight the importance of modulating the excited-state dynamics by tuning the laser frequency. We here note that although we only include the first 4 singlet excited states in our model, the next optically allowed transition is $\sim 0.3 \mathrm{eV}$ higher than the ${ }^{1} \mathrm{MC}$. This leads to the conclusion that in the case of $60 \mathrm{fs}$ pump pulse, the ${ }^{1} \mathrm{MC}$ can be selectively excited, even if higher-lying excited states are included in the model. We mention that the experimental realization of the ${ }^{1} \mathrm{GS} \rightarrow{ }^{1} \mathrm{MC}$ excitation has not yet been reported.

\section{B. Elastic x-ray scattering}

In the following, we utilize the above-discussed quantum dynamics to simulate the TR elastic XDS signal of [Fe(bmip $\left.)_{2}\right]^{2+}$.

First, we calculate the scattering signals for the optimized structures of the ${ }^{1} \mathrm{GS},{ }^{3} \mathrm{MLCT}$, and ${ }^{3} \mathrm{MC}$ states. In Fig. 5, we present the calculated scattering signal for the ${ }^{1} \mathrm{GS}$, as well as the difference XDS signal for the lowest-lying ${ }^{3} \mathrm{MLCT}$ and ${ }^{3} \mathrm{MC}$ states. Note that for the difference signals, we here assume $100 \%$ excitation fraction. As seen in Fig. 5, the scattering signal $\Delta S$ is dominated by the negative feature centered at $\sim 0.5 \AA^{-1}$. This is a well-known characteristic of expansion of the molecule due to elongation of the $\mathrm{Fe}$ ligand bonds, which has been observed experimentally for related transition metal complexes. ${ }^{10,65-67}$ This negative difference signal is assigned to the decrease of electron density within the dimensions

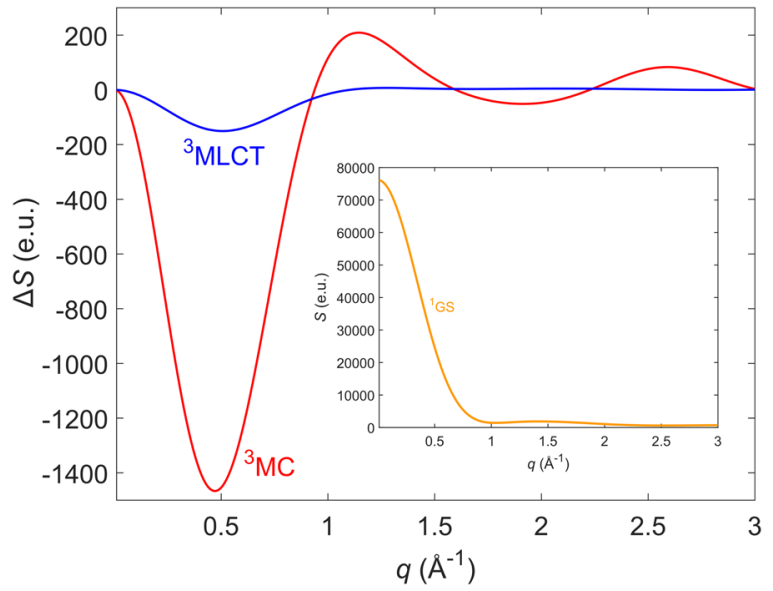

FIG. 5. Difference elastic XDS signal $\Delta S$ for the optimized lowest-lying ${ }^{3} M L C T$ and ${ }^{3} \mathrm{MC}$ structures, relative to the ${ }^{1} \mathrm{GS}$ scattering signal $S$, shown in the inset. The shown difference signals correspond to $100 \%$ excitation fraction. of the molecule. It is also clear from Fig. 5 that $\Delta S$ exhibits significantly larger changes for the optimized ${ }^{3} \mathrm{MC}$, than for the ${ }^{3}$ MLCT structure. This is because the average structural changes for the ${ }^{3} \mathrm{MC}$ states, relative to the ${ }^{1} \mathrm{GS}$, are much larger, $\Delta R_{\mathrm{Fe}-\mathrm{N}}=0.230 \AA$ and $R_{\mathrm{Fe}-\mathrm{C}}=0.077 \AA$, in contrast to $\Delta R_{\mathrm{Fe}-\mathrm{N}}=-0.003 \AA$ and $R_{\mathrm{Fe}-\mathrm{C}}$ $=0.026 \AA$, for the optimized ${ }^{3}$ MLCT. The structural changes in the ${ }^{3} \mathrm{MC}$ state are thus dominated by the $\mathrm{Fe}-\mathrm{N}$ stretching, which is explained by the occupation of the molecular orbital with a strong antibonding $3 d_{z^{2}}$ character (see Fig. 3). The difference scattering is small for the ${ }^{3}$ MLCT states, since the structural changes are small. This is due to the occupation of an orbital with $\mathrm{L}-\pi^{*}$ character that is not involved in the Fe-ligand bonding. The structural distortion in the excited states is also reflected in $\Delta S$ at $q$ above $1 \AA^{-1}$, albeit its amplitude is reduced by one order of magnitude, compared to the low $q$ feature, as seen in Fig. 5. We note that the optimizations of the triplet MLCT and MC states were carried out within unrestricted DFT. This method does not allow practical optimization of the singlet excited states, as they converge to the electronic ground state. However, the structural changes are mainly determined by the electronic character, irrespective of the spin multiplicity. Therefore, very similar difference scattering is expected for the singlet excited states ${ }^{1} \mathrm{MLCT}$ and ${ }^{1} \mathrm{MC}$, to those of the triplet counterparts shown in Fig. 5.

In the following, we discuss the TR difference scattering $\Delta S$, simulated for the 5D QD shown in Fig. 4(b), i.e., the case of excitation by a $60 \mathrm{fs}$ pulse, resonant to the ${ }^{1} \mathrm{GS} \rightarrow{ }^{1}$ MLCT transition. Figure 6 presents the simulated TR- $\Delta S$. In the top panel of Fig. 6 , we observe the growth of the negative low- $q$ difference signal from $\sim 0.5$ ps. Based on the amplitude of the simulated TR- $\Delta S$, this has to correspond to the population of an MC state. The population dynamics shown in Fig. 4(b) identifies that this low- $q$ signal is dominated by the contribution from ${ }^{3} \mathrm{MCs}$. This is consistent with the difference scattering, calculated for the optimized ${ }^{3} \mathrm{MC}$ structure, assuming $100 \%$ excitation fraction, shown in Fig. 5. Note that no ${ }^{1} \mathrm{MC}$ states are populated here, as they are energetically inaccessible from the excited ${ }^{1}$ MLCT states, and thus, they do not contribute to the difference scattering. In the Fig. 6 bottom panel, we removed the low- $q$ region below $0.9 \AA^{-1}$, which enables us to resolve the positive difference signal centered at $\sim 1.1 \AA$. This positive difference signal shows a very similar pattern to the negative low- $q$ counterpart. Both negative and positive difference signals exhibit weak oscillations, which are discussed below.

Figure 7 shows the simulated decomposed TR- $\Delta S$ signal at $q=0.5 \AA^{-1}$ into components for electronic states with different character and multiplicity. This confirms that the difference scattering is dominated by the ${ }^{3} \mathrm{MC}$ contribution. Furthermore, the weak oscillations in $\Delta S$, observed in Fig. 6, also appear in Fig. 7. The oscillations are most important for the ${ }^{3} \mathrm{MC}$ states, as the ${ }^{1,3}$ MLCT difference scattering is very weak and vanishes after $\sim 1 \mathrm{ps}$. Based on the Fourier transform of the ${ }^{3} \mathrm{MC}$ signal, we assign the dominant component of the oscillations to the vibrational motion along $v_{6}$ in the ${ }^{3} \mathrm{MC}$ states. For the interpretation of the observed oscillatory signal, in Fig. 8, we plot the time-dependent $1 \mathrm{D}$ reduced wavepacket density (integrated over all nuclear DOF except $v_{6}$ ) in the ${ }^{3} \mathrm{MC}$ states (weighted by the relative populations of the $9{ }^{3} \mathrm{MC}$ components) along the principal breathing mode $v_{6}$, as well as the temporal evolution of the wavepacket centroid (inset). Based on this figure, we interpret the ${ }^{3} \mathrm{MC}$ wavepacket as vibrationally excited harmonic oscillator 

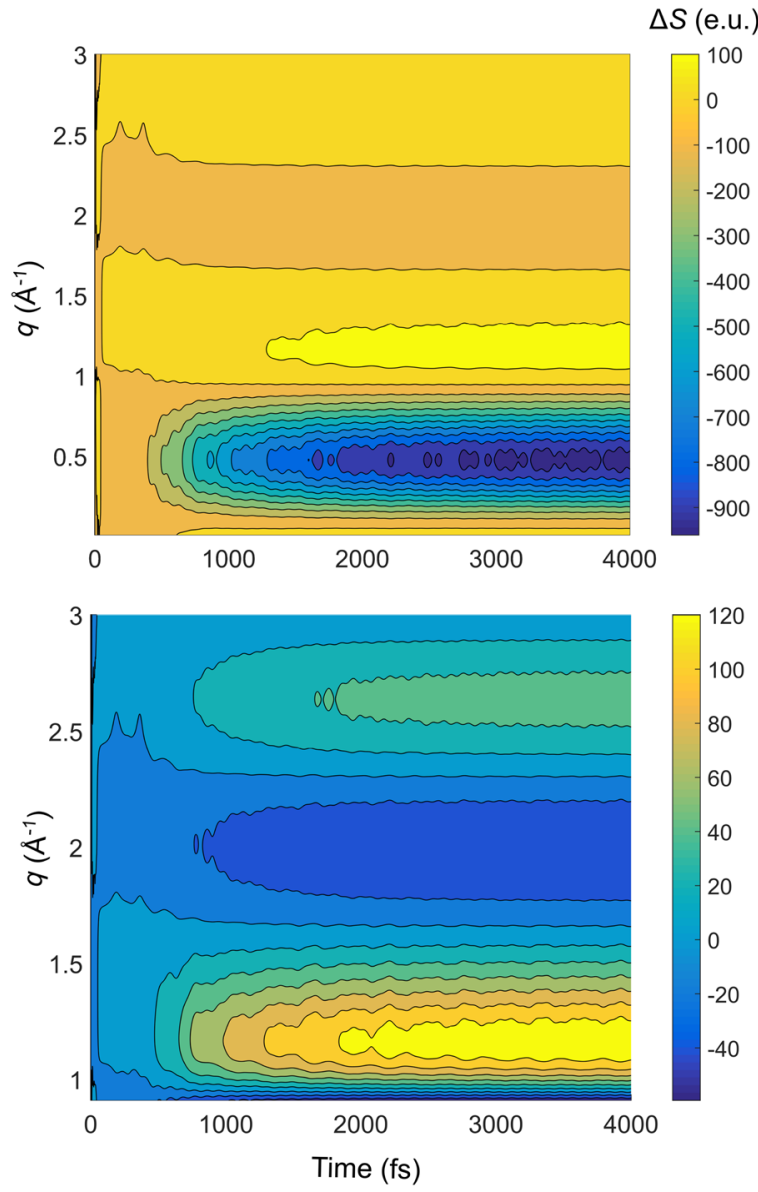

FIG. 6. TR- $\triangle S$ from $5 D$ QD utilizing a 60 fs pump pulse, whose laser frequency is tuned to the ${ }^{1} \mathrm{MLCT}$ resonance [Fig. 4(b)]. Top: $0 \AA^{-1} \leq q \leq 3 \AA^{-1}$, bottom: $0.9 \AA^{-1} \leq q \leq 3 \AA^{-1}$.

eigenstates corresponding to the potential energy at which the wavepacket is created on the ${ }^{3} \mathrm{MC}$ PES. From Fig. 8, it is also apparent that the ${ }^{3} \mathrm{MC}$ wavepacket is almost stationary, which we attribute to very fast dephasing. The ${ }^{3} \mathrm{MC}$ wavepacket thus exhibits a rather small nuclear motion, which is the reason for the weak oscillations observed in the simulated TR-XDS (Fig. 6). A similar scenario is expected for the other two tuning modes $v_{12}$ and $v_{36}$.

We now recall that the XDS signal within our approach is simulated using the centroid of the nuclear wavepacket in each electronic state. We assessed the validity of this approximation by simulating the TR difference scattering by the reduced 1D wavepacket density along the principal mode $v_{6}$. The obtained result (not shown) is in agreement with the one resulting from the $v_{6}$ centroids, which in turn accounts for most of the TR difference scattering shown in Fig. 6. We interpret this result and the validity of our wavepacket centroid approach based on the dependence of $\Delta S$ on the nuclear coordinates. In case of the low- $q\left(q<1.5 \AA^{-1}\right)$ XDS analyzed in this work, the calculated difference scattering is expected to vary linearly with the changes in nuclear coordinates. This is due to the fact that

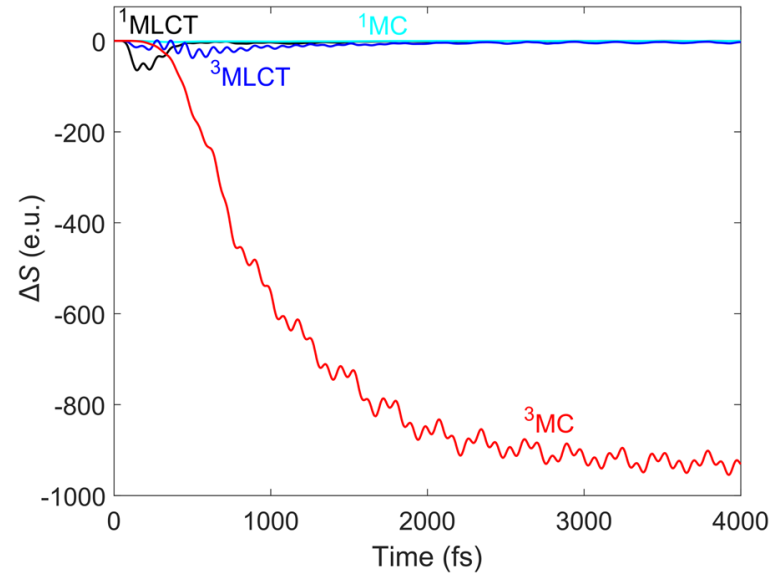

FIG. 7. Decomposition of the TR- $\Delta S$ shown in Fig. 6 at $q=0.5 \AA^{-1}$ for electronic states with different character and multiplicity. These signals are obtained from the QD simulation utilizing a 60 fs ${ }^{1}$ MLCT-resonant pump pulse [see Fig. 4(b)].

in the low- $q$ region, the change in the $\sin \left(q D_{i j}\right) / q D_{i j}$ term of Eq. (18) becomes practically linear with respect to the changes in nuclear coordinates $\Delta D_{i j}{ }^{68}$ The $\Delta S-\Delta D_{i j}$ (or $\Delta S-\Delta Q_{i}$ ) linearity eliminates the effect of the wavepacket width on the difference scattering and thus the wavepacket centroid approximation is adequate at low- $q$. This adequacy was indeed numerically observed in a previous theoretical study. ${ }^{49}$ Furthermore, the approach of solely using the wavepacket centroid was capable of extracting vibrational dynamics from the experimental TR difference scattering signal of a transition metal complex related to $\left[\mathrm{Fe}(\mathrm{bmip})_{2}\right]{ }^{2+} .{ }^{10}$ We note that the centroid approximation might break down in the high- $q$ region, which points toward a possibility of refining the shape of the nuclear wavepacket with access to high- $q$ data. Experimentally, this can become available when the European XFEL reaches its full design parameters and with

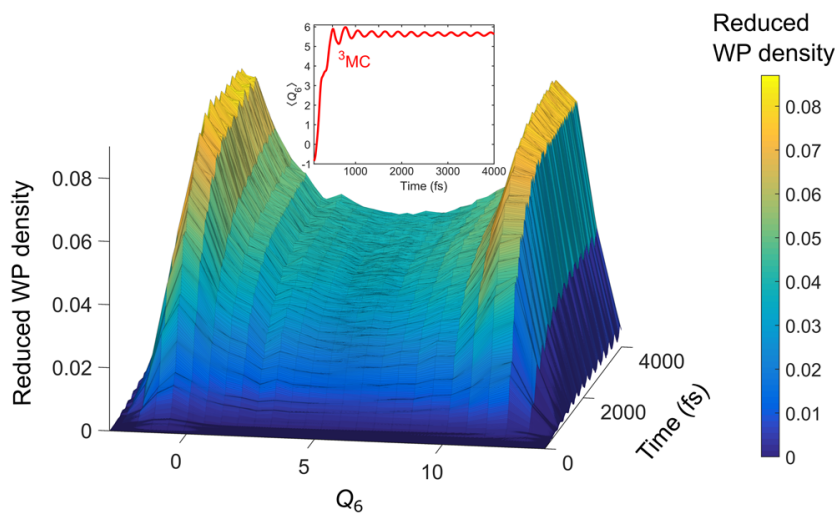

FIG. 8. Time-dependent ${ }^{3} \mathrm{MC}$ reduced wavepacket density along $v_{6}$, obtained from the QD simulation utilizing a $60 \mathrm{fs}^{1} \mathrm{MLCT}$-resonant pump pulse [see Fig. 4(b)]. At $Q_{6}=5, \Delta R_{\mathrm{Fe}-\mathrm{N}}=0.057 \AA$ and $\Delta R_{\mathrm{Fe}-\mathrm{C}}=0.054 \AA$, relative to $Q_{6}=0$; see also Fig. 2(a) for correspondence between $Q_{6}$ and the change in $\mathrm{Fe}-\mathrm{N} / \mathrm{C}$ bond lengths. The inset shows the temporal evolution of the center of the ${ }^{3} \mathrm{MC}$ wavepacket along $v_{6}$. 


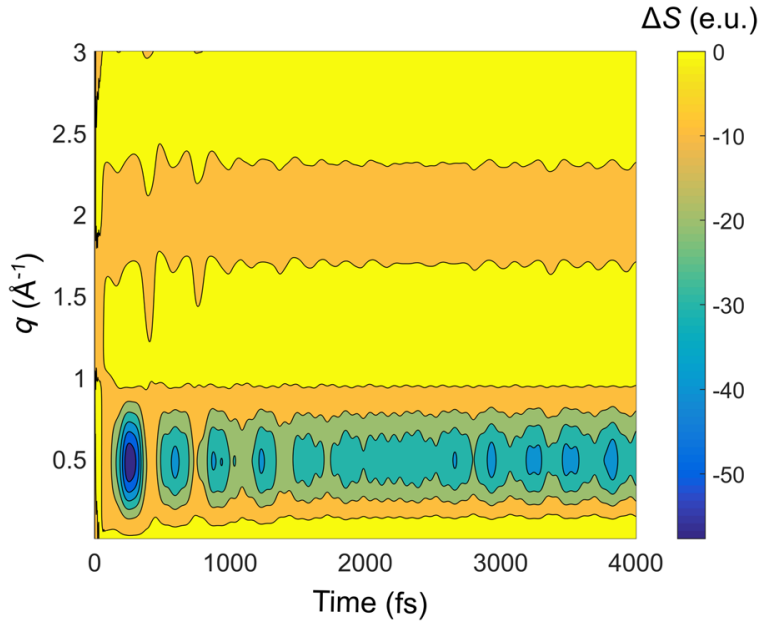

FIG. 9. TR- $\Delta S$ from $5 D$ QD utilizing a 60 fs pump pulse, whose laser frequency is tuned above $0.2 \mathrm{eV}$ the ${ }^{1} \mathrm{MLCT}$ resonance [Fig. 4(d)].

the LCLS (Linac Coherent Light Source) II upgrade, both expected within the next year.

Finally, we investigate the effect of tuning the laser frequency $0.2 \mathrm{eV}$ above the ${ }^{1} \mathrm{MLCT}$ resonance on the simulated difference scattering. Figure 9 presents the resulting TR- $\Delta S$, which is decomposed to components by electronic states in Fig. 10. First, we observe that the difference scattering amplitude is reduced by a factor of $\sim 20$, compared to the ${ }^{1}$ MLCT resonant case shown in Fig. 6. This is because here mainly the ${ }^{1} \mathrm{MC}$ states are excited, which exhibit one order of magnitude smaller oscillator strength from the ${ }^{1} \mathrm{GS}$, relative to the ${ }^{1}$ MLCTs (see Table I), and the same field amplitude $E_{0}$ is applied for all simulations. This weak difference signal can be enhanced by increasing $E_{0}$. Importantly, the difference scattering

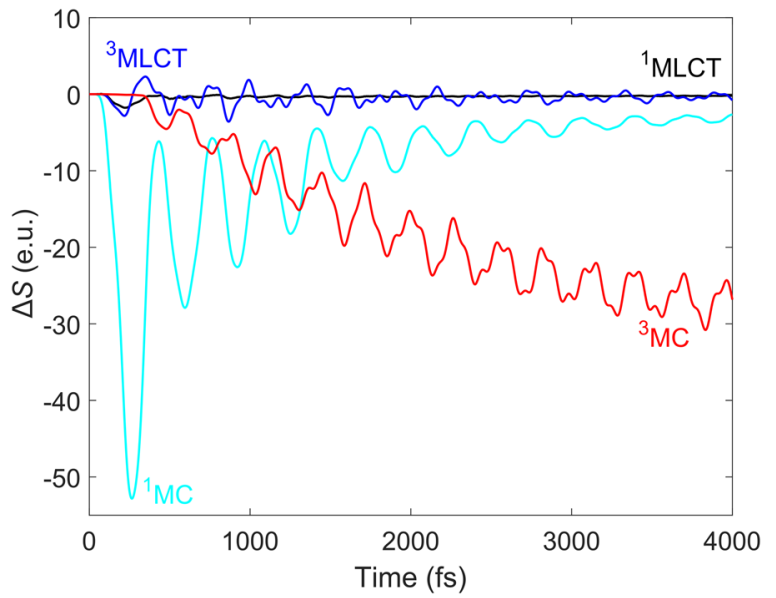

FIG. 10. Decomposition of the TR- $\Delta S$ shown in Fig. 9 at $q=0.5 \AA^{-1}$ for electronic states with different character and multiplicity. These signals are obtained from the QD simulation utilizing a 60 fs pump pulse, tuned $0.2 \mathrm{eV}$ above the ${ }^{1} \mathrm{MLCT}$ resonance [see Fig. 4(d)]. signal rises at early times, i.e., 250 fs and exhibits strong coherent oscillations, whose 330 fs period, as identified by Fourier transformation, matches the vibrational period of the breathing mode $v_{6}$ in the ${ }^{1} \mathrm{MC}$ states. From this and Fig. 10, we assign the early time oscillatory signal to the ${ }^{1} \mathrm{MC}$ states. The large $\Delta S$ amplitudes stem from the large relative ${ }^{1} \mathrm{MC}$ population at early times and the strong vibrational motion along $v_{6}$ (with $\mathrm{Fe}-\mathrm{N}$ and $\mathrm{Fe}-\mathrm{C}$ amplitudes of $\sim 0.1 \AA$; see Fig. 13 in Appendix B), caused by the nonequilibrium excitation of the ${ }^{1} \mathrm{MC}$ states. We illustrate these vibrational dynamics in Fig. 11, in which we plot the ${ }^{1} \mathrm{MC}$ wavepacket centroid along $v_{6}$ in function of time (cyan). The dominance of $v_{6}$ in the nuclear dynamics is confirmed by the oscillations of the timedependent distances, $R_{\mathrm{Fe}-\mathrm{N}}(t)$ and $R_{\mathrm{Fe}-\mathrm{C}}(t)$, obtained by the evolution of wavepacket centroids along all 5 modes (Appendix B, Fig. 13). We recall that the strong vibrational motion along $v_{6}$ is the reason for the ballistic population dynamics shown in Fig. 4(d). Figure 11 reveals that the ${ }^{1} \mathrm{MC}$ wavepacket is strongly nonstationary and dephases on the picosecond time scale. At later times $t>1 \mathrm{ps}$, the ${ }^{1} \mathrm{MC} \Delta S$ signal is significantly reduced, due to the combination of population transfer to the ${ }^{3}$ MLCT and ${ }^{3} \mathrm{MC}$ states [see Fig. 4(d)] and dephasing. At these later times, the ${ }^{3} \mathrm{MC}$ signal dominates, similar to the case of ${ }^{1} \mathrm{MLCT}$-resonant excitation. The ${ }^{3} \mathrm{MC}$ difference scattering displayed in Fig. 10 also exhibits oscillations, dominated by the $v_{6}$ component. The corresponding vibrational dynamics along $v_{6}$ are shown in Fig. 11 (red). Although large-amplitude vibrations occur during the first picosecond, this is only weakly reflected in $\Delta S$, since the ${ }^{3} \mathrm{MC}$ population is relatively small during these times, as seen in Fig. 4(d). Later, the ${ }^{3} \mathrm{MC}$ population is dominant; however, the ${ }^{3} \mathrm{MC}$ wavepacket undergoes dephasing leading to moderateamplitude oscillations in the simulated difference scattering signal. Still, these oscillations are more prominent than those observed for the ${ }^{1}$ MLCT-resonant excitation. This is due to the fact that the ${ }^{3} \mathrm{MC}$ wavepacket is nonstationary, preserving some of its coherence

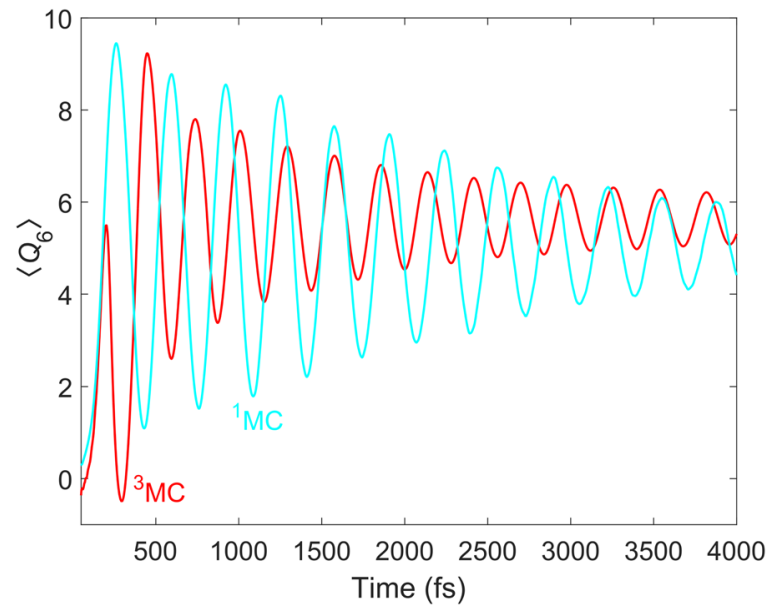

FIG. 11. Temporal evolution of the center $\left\langle Q_{6}\right\rangle$ of the ${ }^{1} \mathrm{MC}$ and ${ }^{3} \mathrm{MC}$ wavepackets (weighted by the corresponding relative ${ }^{1} \mathrm{MC}$ or ${ }^{3} \mathrm{MC}$ populations), as obtained from the QD simulation employing a $60 \mathrm{fs}$ pump pulse, whose laser frequency is tuned $0.2 \mathrm{eV}$ above the ${ }^{1} \mathrm{MLCT}$ resonance [see Fig. $4(\mathrm{~d})$ ]. At $Q_{6}=5, \Delta R_{\mathrm{Fe}-\mathrm{N}}$ $=0.057 \AA$ and $\Delta R_{\mathrm{Fe}-\mathrm{C}}=0.054 \AA$, relative to $Q_{6}=0$; see also Fig. 2(a) for correspondence between $Q_{6}$ and the change in $\mathrm{Fe}-\mathrm{N} / \mathrm{C}$ bond lengths. 
originating from the ${ }^{1} \mathrm{MC}$ wavepacket, created in a nonequilibrium region. We close our discussion by noting that vibrational dynamics of other photoactivated transition metal complexes have also been observed experimentally by elastic TR-XDS. ${ }^{65,68,69}$

\section{CONCLUDING REMARKS AND OUTLOOK}

In the present work, we simulated the excited-state population dynamics and the difference elastic XDS signal of $\left[\mathrm{Fe}(\mathrm{bmip})_{2}\right]^{2+}$ excited into ${ }^{1} \mathrm{MLCT}$ and ${ }^{1} \mathrm{MC}$ states. In the case of ${ }^{1} \mathrm{MLCT}$-resonant excitation, only the ${ }^{1}$ MLCT states are populated by the pump pulse, independent of the pulse duration. This excitation process takes place at an equilibrium position, leading to weak nuclear dynamics in the excited-state manifold and thus the kinetic population flow. The simulated TR difference XDS signal $\Delta S$ is dominated by the low- $q\left(\sim 0.5 \AA^{-1}\right)$ component assigned to ${ }^{3} \mathrm{MC}$ states, for which the structural changes, i.e., the elongation of $\mathrm{Fe}-\mathrm{N} / \mathrm{C}$ bonds, are much larger than those for the MLCTs. The ${ }^{1} \mathrm{MC}$ states would also have a strong $\Delta S$ signal; however, these states are not excited by the pump pulse and are inaccessible from the MLCTs. Therefore, the ${ }^{1} \mathrm{MC}$ difference signal is absent in this case. The TR- $\Delta S$ exhibits only small-amplitude oscillations, as only weak vibrational dynamics occur in the ${ }^{3} \mathrm{MC}$ states. This is interpreted by the stationary ${ }^{3} \mathrm{MC}$ wavepacket, created by the kinetic population flow from the ${ }^{3}$ MLCT states near the FC geometry.

Tuning the laser frequency $0.2 \mathrm{eV}$ above the ${ }^{1} \mathrm{MLCT}$ resonance, the excitation fraction of ${ }^{1} \mathrm{MLCT}$ and ${ }^{1} \mathrm{MC}$ states can be controlled by the pulse duration. While for the short $10 \mathrm{fs}$ pulse, both states are excited, the ${ }^{1} \mathrm{MC}$ states can be selectively excited by utilization of a longer $60 \mathrm{fs}$ pump pulse. In both cases, the ${ }^{1} \mathrm{MC}$ wavepacket is created at a nonequlibrium region along the principal mode $v_{6}$, i.e., far from the minimum on the ${ }^{1} \mathrm{MC}$ PES. This induces a strong nuclear motion, leading to ballistic dynamics, when the ${ }^{1} \mathrm{MC}$ wavepacket reaches an intersection between ${ }^{1} \mathrm{MC}$ and ${ }^{1,3} \mathrm{MLCT}$ PESs. These prominent vibrational dynamics are clearly reflected in the coherent oscillations in the TR difference scattering during the first picosecond. These oscillations occur with a dominant period of $\sim 330 \mathrm{fs}$, matching the vibrational period of breathing mode $v_{6}$ in the ${ }^{1} \mathrm{MC}$ states. These results identify $v_{6}$ as the principal mode and evidence the importance of controlling excited-state dynamics by detuning. The ${ }^{1} \mathrm{MC}$ coherent oscillations in TR- $\Delta S$ are damped during the next picoseconds, which is the consequence of the flow of the ${ }^{1} \mathrm{MC}$ population into the MLCTs and dephasing of the nonstationary ${ }^{1} \mathrm{MC}$ wavepacket. Finally, the difference scattering is governed by the ${ }^{3} \mathrm{MC}$ components, due to the population of these states via the MLCTs. As the coherence of the nuclear wavepacket is partially transferred into the ${ }^{3} \mathrm{MC}$ states, the oscillations also appear in the ${ }^{3} \mathrm{MC}$ difference signal, albeit with smaller amplitudes, due to dephasing.

From an X-ray scattering point of view, the simulations reported in this work are an important step for the analysis of TR-XDS experiments. In particular, the decomposition of the overall signal into components by electronic states is very valuable, as this is not accessible from elastic XDS experiments. Based on a recent computational study, ${ }^{56}$ the solute dynamics of the investigated $\left[\mathrm{Fe}(\text { bmip })_{2}\right]^{2+}$ complex should not be affected by a polar solvent, such as acetonitrile. However, this is clearly not true for other molecules, ${ }^{56,70,71}$ which thus require the inclusion of the solvent, even for simulating the solute difference scattering. In addition, the electrons of the solvent also contribute to the difference XDS signal leading to the cage and solvent heating terms. Coupling of the solute quantum dynamics with solvation dynamics ${ }^{70}$ will therefore be a high-priority goal for future studies that will facilitate the analysis of ultrafast XDS experiments.

\section{ACKNOWLEDGMENTS}

The research leading to the presented results has received funding from the Danish Council for Independent Research, Grant No. 4002-00272, the Independent Research Fund Denmark, Grant No. 8021-00347B, and was also supported by the Government of Hungary and the European Regional Development Fund under Grant No. VEKOP-2.3.2-16-2017-00015. The authors are grateful to Asmus O. Dohn and Kristoffer Haldrup for discussions.
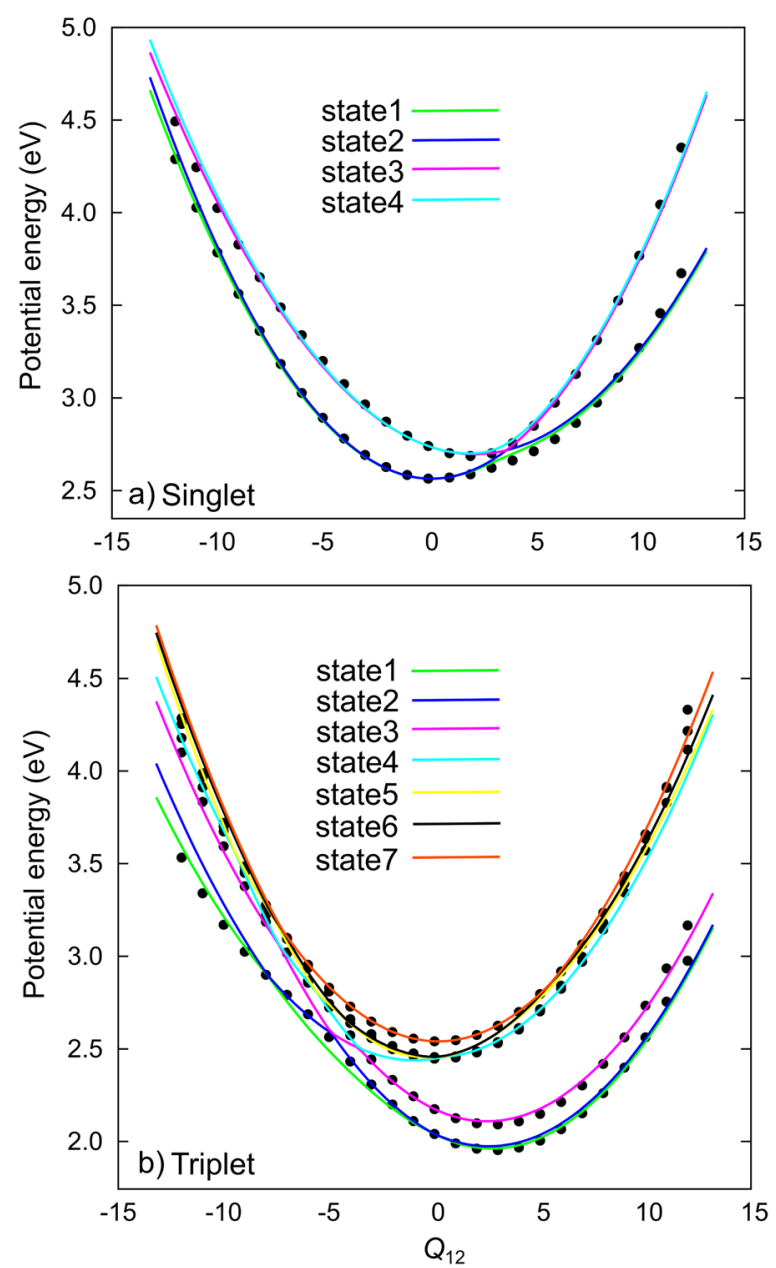

FIG. 12. Adiabatic PESs along mode $v_{12}$. The points display energies computed by TD-DFT at the FC point and geometries distorted along $v_{12}$. The lines represent the fits, obtained by diagonalization of the diabatic Hamiltonian. Nuclear displacements are given in the dimensionless mass-frequency weighted normal coordinate $Q_{12}$ 
TABLE II. Computational details of the QD simulations. $N_{i}$ is the number of primitive harmonic oscillator basis functions used for each mode. $n_{\alpha}$ are the number of single particle functions used to describe the wavepacket in each state.

\begin{tabular}{lcc}
\hline \hline Modes & $N_{i}$ & $n_{\mathrm{S}_{0}}, n_{\mathrm{S}_{1}}, n_{\mathrm{S}_{2}}, n_{\mathrm{S}_{3}}, n_{\mathrm{S}_{4}}, n_{\mathrm{T}_{1}(-1,0,1)}, n_{\mathrm{T}_{2}(-1,0,1)}, n_{\mathrm{T}_{3}(-1,0,1)}, n_{\mathrm{T}_{4}(-1,0,1)}, n_{\mathrm{T}_{5}(-1,0,1)}, n_{\mathrm{T}_{6}(-1,0,1)}, n_{\mathrm{T}_{7}(-1,0,1)}$ \\
\hline$v_{6}$ & 121 & $10,10,10,10,10,10,35,35,10,35,35,30,25,25,12,10,10,10,10,10,10,10,10,10,10,10$ \\
$v_{11}$ & 61 & $10,10,10,10,10,10,25,25,10,12,12,10,10,10,10,10,10,10,10,10,10,10,10,10,10,10$ \\
$v_{12}$ & 61 & $10,10,10,8,8,10,25,25,10,25,25,18,18,18,10,10,10,10,10,10,10,10,10,10,10,10$ \\
$v_{25}$ & 71 & $10,10,10,5,5,10,10,10,10,10,10,10,10,10,10,10,10,10,10,10,10,10,10,10,10,10$ \\
$v_{36}$ & 71 & $10,10,10,10,10,10,12,12,10,12,12,10,10,10,10,10,10,10,10,10,10,10,10,10,10,10$ \\
\hline
\end{tabular}

\section{APPENDIX A: HAMILTONIAN PARAMETERS FOR MODE $v_{12}$}

The vibrational frequency of mode $v_{12}$, as calculated at the

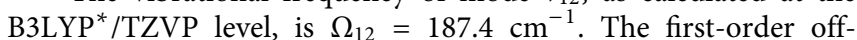
diagonal coefficient (to be multiplied by the corresponding value of $Q_{12}$ to yield the linear diabatic coupling) for singlet states is $\lambda_{\mathrm{S}_{1}, \mathrm{~S}_{3}}=\lambda_{\mathrm{S}_{1}, \mathrm{~S}_{4}}=\lambda_{\mathrm{S}_{2}, \mathrm{~S}_{3}}=\lambda_{\mathrm{S}_{2}, \mathrm{~S}_{4}}=0.004 \mathrm{eV}$; for triplet states, it is $\lambda_{\mathrm{T}_{1}, \mathrm{~T}_{5}}=\lambda_{\mathrm{T}_{1}, \mathrm{~T}_{6}}=\lambda_{\mathrm{T}_{2}, \mathrm{~T}_{5}}=\lambda_{\mathrm{T}_{2}, \mathrm{~T}_{6}}=0.012 \mathrm{eV}$. The linear diabatic coupling between all other states along $v_{12}$ is zero. The diagonal first- $\left(\kappa_{\alpha}\right)$ and second-order $\left(\gamma_{\alpha}\right)$ coefficients are tabulated in Table III (to be multiplied by $Q_{12}$ and $Q_{12}^{2} / 2$, respectively, to yield the diagonal Hamiltonian matrix elements). The Hamiltonian parameters for the other 4 modes are given in Ref. 34. The fits to adiabatic TD-DFT PESs are shown in Fig. 12.

\section{APPENDIX B: Fe-N AND Fe-C VIBRATIONAL DYNAMICS IN THE MC STATES}

The nuclear dynamics is the strongest for the MC states of the detuned simulation. For these MCs, we report the time-dependent $\mathrm{Fe}-\mathrm{N}$ and $\mathrm{Fe}-\mathrm{C}$ distances, as calculated from the wavepacket centroids along all 5 nuclear DOF, weighted by the relative populations

TABLE III. Diagonal first- $\left(\kappa_{\alpha}\right)$ and second-order $\left(\gamma_{\alpha}\right)$ Hamiltonian coefficients for $v_{12}$.

\begin{tabular}{lrr}
\hline \hline$|\alpha\rangle$ & $\kappa_{\alpha}(\mathrm{eV})$ & $\gamma_{\alpha}(\mathrm{eV})$ \\
\hline $\mathrm{S}_{1}$ & -0.004 & 0.001 \\
$\mathrm{~S}_{2}$ & -0.004 & 0.001 \\
$\mathrm{~S}_{3}$ & -0.040 & -0.005 \\
$\mathrm{~S}_{4}$ & -0.040 & -0.005 \\
\hline $\mathrm{T}_{1}$ & -0.055 & -0.002 \\
$\mathrm{~T}_{2}$ & -0.055 & -0.002 \\
$\mathrm{~T}_{3}$ & -0.053 & -0.002 \\
$\mathrm{~T}_{4}$ & 0.018 & -0.004 \\
$\mathrm{~T}_{5}$ & 0.003 & -0.002 \\
$\mathrm{~T}_{6}$ & 0.003 & -0.002 \\
$\mathrm{~T}_{7}$ & -0.004 & -0.001 \\
\hline \hline
\end{tabular}

of the ${ }^{1} \mathrm{MC}$ or ${ }^{3} \mathrm{MC}$ components, as shown in Fig. 13. These dynamics are dominated by the $v_{6}$ vibrational motion $(330 / 285 \mathrm{fs}$ period for $\left.{ }^{1} \mathrm{MC} /{ }^{3} \mathrm{MC}\right)$, and a minor component arises due to $v_{12}$ vibrations (200/190 fs period).
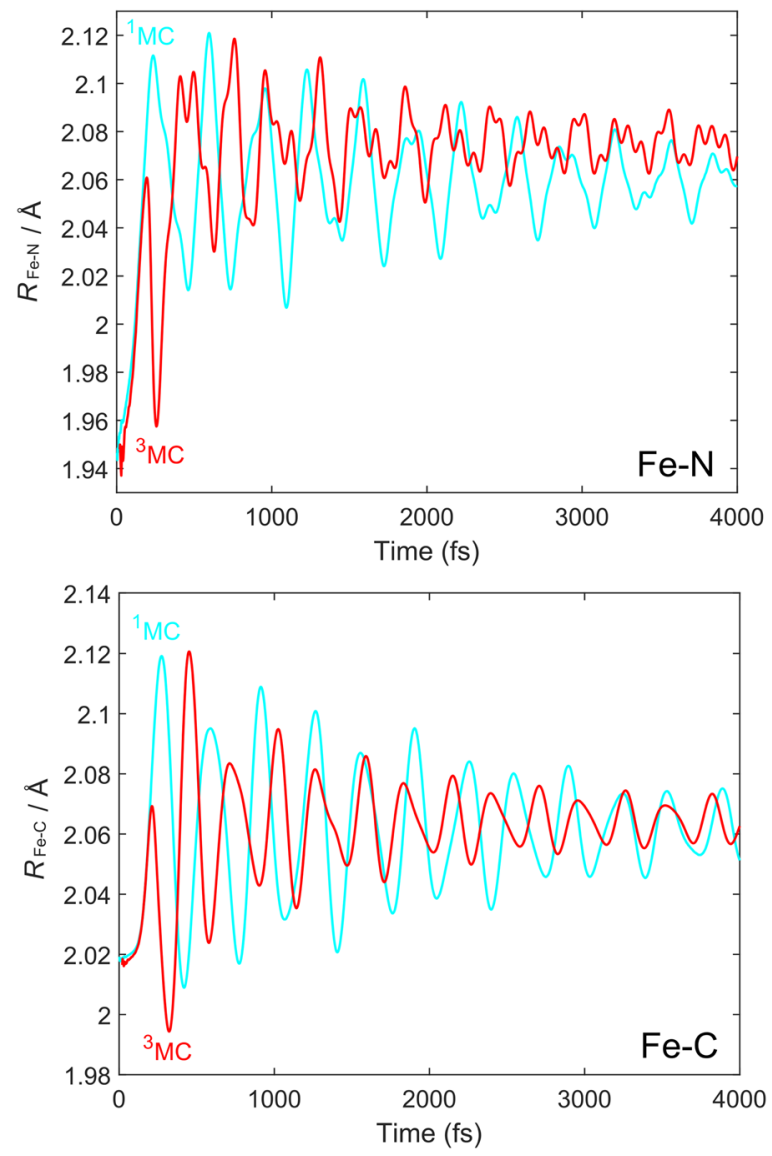

FIG. 13. Temporal evolution of the $\mathrm{Fe}-\mathrm{N}$ (top) and $\mathrm{Fe}-\mathrm{C}$ (bottom) distances for the ${ }^{1} \mathrm{MC}$ and ${ }^{3} \mathrm{MC}$ states of the detuned simulation. The shown $\mathrm{Fe}-\mathrm{N} / \mathrm{Fe}-\mathrm{C}$ values were calculated from the time-dependent wavepacket centroids along all 5 modes, weighted by the relative electronic populations of the ${ }^{1} \mathrm{MC} /{ }^{3} \mathrm{MC}$ components. 


\section{REFERENCES}

${ }^{1}$ B. Lasorne, G. A. Worth, and M. A. Robb, Wiley Interdiscip. Rev.: Comput. Mol. Sci. 1, 460 (2011).

${ }^{2}$ M. Grätzel, J. Photochem. Photobiol. C 4, 145 (2003).

${ }^{3}$ F. Natterer, K. Yang, W. Paul, P. Willke, T. Choi, T. Greber, A. Heinrich, and C. Lutz, Nature 543, 226 (2017).

${ }^{4}$ J. K. McCusker, Science 363, 484 (2019).

${ }^{5}$ C. E. Crespo-Hernndez, B. Cohen, P. M. Hare, and B. Kohler, Chem. Rev. 104, 1977 (2004).

${ }^{6}$ M. Chergui, Acc. Chem. Res. 48, 801 (2015).

${ }^{7}$ T. J. Penfold, E. Gindensperger, C. Daniel, and C. M. Marian, Chem. Rev. 118, 6975 (2018).

${ }^{8}$ T. I. Sølling and K. B. Møller, Struct. Dyn. 5, 060901 (2018).

${ }^{9}$ C. Pellegrini, Eur. Phys. J. H 37, 659 (2012).

${ }^{10}$ E. Biasin, T. B. van Driel, K. S. Kjær, A. O. Dohn, M. Christensen, T. Harlang, P. Chabera, Y. Liu, J. Uhlig, M. Pápai, Z. Németh, R. Hartsock, W. Liang, J. Zhang, R. Alonso-Mori, M. Chollet, J. M. Glownia, S. Nelson, D. Sokaras, T. A. Assefa, A. Britz, A. Galler, W. Gawelda, C. Bressler, K. J. Gaffney, H. T. Lemke, K. B. Møller, M. M. Nielsen, V. Sundström, G. Vankó, K. Wärnmark, S. E. Canton, and K. Haldrup, Phys. Rev. Lett. 117, 013002 (2016).

${ }^{11}$ S. E. Canton, K. S. Kjaer, G. Vanko, T. B. van Driel, S.-i. Adachi, A. Bordage, C. Bressler, P. Chabera, M. Christensen, A. O. Dohn, A. Galler, W. Gawelda, D. Gosztola, K. Haldrup, T. Harlang, Y. Liu, K. B. Moller, Z. Nemeth, S. Nozawa, M. Papai, T. Sato, T. Sato, K. Suarez-Alcantara, T. Togashi, K. Tono, J. Uhlig, D. A. Vithanage, K. Warnmark, M. Yabashi, J. Zhang, V. Sundstrom, and M. M. Nielsen, Nat. Commun. 6, 6359 (2015).

${ }^{12}$ T. J. Penfold, M. Pápai, T. Rozgonyi, K. B. Møller, and G. Vankó, Faraday Discuss. 194, 731 (2016).

${ }^{13}$ S. P. Neville, V. Averbukh, M. Ruberti, R. Yun, S. Patchkovskii, M. Chergui, A. Stolow, and M. S. Schuurman, J. Chem. Phys. 145, 144307 (2016).

${ }^{14}$ S. P. Neville, M. Chergui, A. Stolow, and M. S. Schuurman, Phys. Rev. Lett. 120, 243001 (2018)

${ }^{15}$ T. Northey, J. Duffield, and T. J. Penfold, J. Chem. Phys. 149, 124107 (2018).

${ }^{16}$ T. Northey, J. Norell, A. E. A. Fouda, N. A. Besley, M. Odelius, and T. J. Penfold, "Ultrafast nonadiabatic dynamics probed by nitrogen K-edge absorption spectroscopy," Phys. Chem. Chem. Phys. (published online).

${ }^{17}$ S. Tsuru, M. L. Vidal, M. Pápai, A. I. Krylov, K. B. Møller, and S. Coriani, "Time-resolved near-edge X-ray absorption fine structure of pyrazine fromelectronic structure and nuclear wave packet dynamics simulations," J. Chem. Phys. (to be published).

${ }^{18}$ A. Debnarova, S. Techert, and S. Schmatz, J. Chem. Phys. 125, 224101 (2006).

${ }^{19}$ M. Ben-Nun, J. Cao, and K. R. Wilson, J. Phys. Chem. A 101, 8743 (1997).

${ }^{20}$ U. Lorenz, K. B. Møller, and N. E. Henriksen, Phys. Rev. A 81, 023422 (2010).

${ }^{21}$ A. Kirrander, K. Saita, and D. V. Shalashilin, J. Chem. Theory Comput. 12, 957 (2016).

${ }^{22}$ A. Moreno Carrascosa, H. Yong, D. L. Crittenden, P. M. Weber, and A. Kirrander, J. Chem. Theory Comput. 15, 2836 (2019).

${ }^{23}$ M. Simmermacher, N. E. Henriksen, K. B. Møller, A. Moreno Carrascosa, and A. Kirrander, Phys. Rev. Lett. 122, 073003 (2019).

${ }^{24}$ G. Dixit and R. Santra, Phys. Rev. A 96, 053413 (2017).

${ }^{25}$ M. Kowalewski, K. Bennett, and S. Mukamel, Struct. Dyn. 4, 054101 (2017).

${ }^{26}$ K. Bennett, M. Kowalewski, J. R. Rouxel, and S. Mukamel, Proc. Natl. Acad. Sci. U. S. A. 115, 6538 (2018).

${ }^{27}$ Y. Liu, T. Harlang, S. E. Canton, P. Chábera, K. Suárez-Alcántara, A. Fleckhaus, D. A. Vithanage, E. Göransson, A. Corani, R. Lomoth, V. Sundström, and K. Wärnmark, Chem. Commun. 49, 6412 (2013).

${ }^{28}$ Y. Liu, P. Persson, V. Sundström, and K. Wärnmark, Acc. Chem. Res. 49, 1477 (2016).

${ }^{29}$ Y. Liu, K. S. Kjær, L. A. Fredin, P. Chábera, T. Harlang, S. E. Canton, S. Lidin, J. Zhang, R. Lomoth, K.-E. Bergquist, P. Persson, K. Wärnmark, and V. Sundström, Chem. Eur. J. 21, 3628 (2015).
${ }^{30}$ T. Harlang, Y. Liu, O. Gordivska, L. Fredin, C. Ponseca, P. Huang, P. Chábera, K. Kjaer, H. Mateos, J. Uhlig, R. Lomoth, R. Wallenberg, S. Styring, P. Persson, V. Sundström, and K. Wärnmark, Nat. Chem. 7, 883 (2015).

${ }^{31}$ T. Duchanois, T. Etienne, C. Cebrián, L. Liu, A. Monari, M. Beley, X. Assfeld, S. Haacke, and P. C. Gros, Eur. J. Inorg. Chem. 2015, 2469.

${ }^{32}$ L. Liu, T. Duchanois, T. Etienne, A. Monari, M. Beley, X. Assfeld, S. Haacke, and P. C. Gros, Phys. Chem. Chem. Phys. 18, 12550 (2016).

${ }^{33}$ D. Leshchev, T. Harlang, L. Fredin, D. Khakhulin, Y. Liu, E. Biasin, M. Laursen, G. Newby, K. Haldrup, M. Nielsen, K. Wärnmark, V. Sundström, P. Persson, K. Kjær, and M. Wulff, Chem. Sci. 9, 405 (2018).

${ }^{34}$ M. Pápai, G. Vankó, T. Rozgonyi, and T. J. Penfold, J. Phys. Chem. Lett. 7, 2009 (2016).

${ }^{35}$ M. Pápai, M. Simmermacher, T. J. Penfold, K. B. Møller, and T. Rozgonyi, J. Chem. Theory Comput. 14, 3967 (2018).

${ }^{36}$ H.-D. Meyer, U. Manthe, and L. Cederbaum, Chem. Phys. Lett. 165, 73 (1990).

${ }^{37}$ M. Beck, A. Jäckle, G. Worth, and H.-D. Meyer, Phys. Rep. 324, 1 (2000).

${ }^{38}$ H.-D. Meyer, F. Gatti, and G. Worth, Multidimensional Quantum Dynamics: MCTDH Theory and Applications (John Wiley \& Sons, 2009), pp. 1-419.

${ }^{39}$ L. S. Cederbaum, H. Köppel, and W. Domcke, Int. J. Quantum Chem. 20, 251 (1981).

${ }^{40} \mathrm{H}$. Köppel, W. Domcke, and L. S. Cederbaum, "Multimode molecular dynamics beyond the Born-Oppenheimer approximation," in Advances in Chemical Physics (John Wiley \& Sons, 1984), Chap. 57, pp. 59-246.

${ }^{41}$ T. J. Penfold and G. A. Worth, J. Chem. Phys. 131, 064303 (2009).

${ }^{42}$ G. Capano, M. Chergui, U. Rothlisberger, I. Tavernelli, and T. J. Penfold, J. Phys. Chem. A 118, 9861 (2014).

${ }^{43}$ J. Eng, C. Gourlaouen, E. Gindensperger, and C. Daniel, Acc. Chem. Res. 48, 809 (2015).

${ }^{44}$ M. Pápai, T. J. Penfold, and K. B. Møller, J. Phys. Chem. C 120, 17234 (2016).

${ }^{45}$ M. Fumanal, E. Gindensperger, and C. Daniel, J. Chem. Theory Comput. 13, 1293 (2017).

${ }^{46}$ M. Fumanal, E. Gindensperger, and C. Daniel, J. Phys. Chem. Lett. 9, 5189 (2018).

${ }^{47}$ K. B. Møller and N. E. Henriksen, "Time-resolved x-ray diffraction: The dynamics of the chemical bond," in Molecular Electronic Structures of Transition Metal Complexes I. Structure and Bonding, Vol. 142, edited by D. Mingos, P. Day, and J. Dahl (Springer, Berlin, Heidelberg, 2011), pp. 185-211.

${ }^{48}$ E. Biasin, T. B. van Driel, G. Levi, M. G. Laursen, A. O. Dohn, A. Moltke, P. Vester, F. B. K. Hansen, K. S. Kjaer, T. Harlang, R. Hartsock, M. Christensen, K. J. Gaffney, N. E. Henriksen, K. B. Møller, K. Haldrup, and M. M. Nielsen, J. Synchrotron Rad. 25, 306 (2018).

${ }^{49}$ A. O. Dohn, E. Biasin, K. Haldrup, M. M. Nielsen, N. E. Henriksen, and K. B. Møller, J. Phys. B: At., Mol. Opt. Phys. 48, 244010 (2015).

${ }^{50}$ S. Hirata and M. Head-Gordon, Chem. Phys. Lett. 314, 291 (1999).

${ }^{51}$ M. Reiher, O. Salomon, and B. Artur Hess, Theor. Chem. Acc. 107, 48 (2001).

${ }^{52}$ A. Schäfer, C. Huber, and R. Ahlrichs, J. Chem. Phys. 100, 5829 (1994).

${ }^{53}$ F. Neese, Wiley Interdiscip. Rev.: Comput. Mol. Sci. 2, 73 (2012).

${ }^{54}$ F. Neese, J. Comput. Chem. 24, 1740 (2003).

${ }^{55}$ F. Neese, F. Wennmohs, A. Hansen, and U. Becker, Chem. Phys. 356, 98 (2009).

${ }^{56}$ M. Pápai, M. Abedi, G. Levi, E. Biasin, M. M. Nielsen, and K. B. Møller, J. Phys. Chem. C 123, 2056 (2019).

${ }^{57}$ F. Gatti, B. Lasorne, H.-D. Meyer, and A. Nauts, Applications of Quantum Dynamics in Chemistry, Lecture Notes in Chemistry Vol 98 (Springer International Publishing, 2017).

${ }^{58}$ F. Wang and T. Ziegler, J. Chem. Phys. 123, 154102 (2005).

${ }^{59}$ E. van Lenthe, E. J. Baerends, and J. G. Snijders, J. Chem. Phys. 99, 4597 (1993). ${ }^{60}$ G. te Velde, F. M. Bickelhaupt, E. J. Baerends, C. Fonseca Guerra, S. J. A. van Gisbergen, J. G. Snijders, and T. Ziegler, J. Comput. Chem. 22, 931 (2001).

${ }^{61}$ MATLAB and Statistics Toolbox Release 2019a, The MathWorks, Inc., Natick, MA, USA.

${ }^{62}$ See http://lampx.tugraz.at/ hadley/ss1/crystaldiffraction/atomicformfactors/fo rmfactors.php for the utilized Cromer-Mann coefficients.

${ }^{63}$ T. Penfold, M. Pápai, K. Møller, and G. Worth, Comput. Theor. Chem. 1160, 24 (2019). 
${ }^{64}$ R. Monni, G. Capano, G. Auböck, H. B. Gray, A. Vlček, I. Tavernelli, and M. Chergui, Proc. Natl. Acad. Sci. U. S. A. 115, E6396 (2018).

${ }^{65}$ K. S. Kjær, T. B. Van Driel, T. C. B. Harlang, K. Kunnus, E. Biasin, K. Ledbetter, R. W. Hartsock, M. E. Reinhard, S. Koroidov, L. Li, M. G. Laursen, F. B. Hansen, P. Vester, M. Christensen, K. Haldrup, M. M. Nielsen, A. O. Dohn, M. Pápai, K. B. Møller, P. Chabera, Y. Liu, H. Tatsuno, C. Timm, M. Jarenmark, J. Uhlig, V. Sundström, K. Wärnmark, P. Persson, Z. Németh, D. S. Szemes, É. Bajnóczi, G. Vankó, R. Alonso-Mori, J. M. Glownia, S. Nelson, M. Sikorski, D. Sokaras, S. E. Canton, H. T. Lemke, and K. J. Gaffney, Chem. Sci. 10, 5749 (2019).

${ }^{66}$ K. Haldrup, G. Vankó, W. Gawelda, A. Galler, G. Doumy, A. M. March, E. P. Kanter, A. Bordage, A. Dohn, T. B. van Driel, K. S. Kjr, H. T. Lemke, S. E. Canton, J. Uhlig, V. Sundström, L. Young, S. H. Southworth, M. M. Nielsen, and C. Bressler, J. Phys. Chem. A 116, 9878 (2012).

${ }^{67}$ K. Haldrup, W. Gawelda, R. Abela, R. Alonso-Mori, U. Bergmann, A. Bordage, M. Cammarata, S. E. Canton, A. O. Dohn, T. B. van Driel, D. M. Fritz, A. Galler, P. Glatzel, T. Harlang, K. S. Kjær, H. T. Lemke, K. B. Møller, Z. Németh, M. Pápai, N. Sas, J. Uhlig, D. Zhu, G. Vankó, V. Sundström, M. M. Nielsen, and C. Bressler, J. Phys. Chem. B 120, 1158 (2016).
${ }^{68}$ T. Van Driel, K. Kjær, R. Hartsock, A. Dohn, T. Harlang, M. Chollet, M. Christensen, W. Gawelda, N. Henriksen, J. Kim, K. Haldrup, K. Kim, H. Ihee, J. Kim, H. Lemke, Z. Sun, V. Sundström, W. Zhang, D. Zhu, K. Møller, M. Nielsen, and K. Gaffney, Nat. Commun. 7, 13678 (2016).

${ }^{69}$ K. Haldrup, G. Levi, E. Biasin, P. Vester, M. G. Laursen, F. Beyer, K. S. Kjær, T. Brandt van Driel, T. Harlang, A. O. Dohn, R. J. Hartsock, S. Nelson, J. M. Glownia, H. T. Lemke, M. Christensen, K. J. Gaffney, N. E. Henriksen, K. B. Møller, and M. M. Nielsen, Phys. Rev. Lett. 122, 063001 (2019).

${ }^{70}$ J. Cerezo, Y. Liu, N. Lin, X. Zhao, R. Improta, and F. Santoro, J. Chem. Theory Comput. 14, 820 (2018).

${ }^{71}$ K. S. Kjær, K. Kunnus, T. C. B. Harlang, T. B. Van Driel, K. Ledbetter, R. W. Hartsock, M. E. Reinhard, S. Koroidov, L. Li, M. G. Laursen, E. Biasin, F. B. Hansen, P. Vester, M. Christensen, K. Haldrup, M. M. Nielsen, P. Chabera, Y. Liu, H. Tatsuno, C. Timm, J. Uhlig, V. Sundström, Z. Németh, D. S. Szemes, E. Bajnóczi, G. Vankó, R. Alonso-Mori, J. M. Glownia, S. Nelson, M. Sikorski, D. Sokaras, H. T. Lemke, S. E. Canton, K. Wärnmark, P. Persson, A. A. Cordones, and K. J. Gaffney, Phys. Chem. Chem. Phys. 20, 4238 (2018). 